\title{
Advances of Clar's Aromatic Sextet Theory and Randic's Conjugated Circuit Model
}

\author{
Fuji Zhang*,a ${ }^{*}$ Xiaofeng Guo and Heping Zhang ${ }^{\mathrm{b}}$ \\ ${ }^{a}$ School of Mathematical Sciences, Xiamen University, Xiamen, Fujian 361005, China \\ ${ }^{b}$ School of Mathematics and Statistics, Lanzhou University, Lanzhou, Gansu 730000, China
}

\begin{abstract}
Clar's aromatic sextet theory provides a good means to describe the aromaticity of benzenoid hydrocarbons, which was mainly based on experimental observations. Clar defined sextet pattern and Clar number of benzenoid hydrocarbons, and he observed that for isomeric benzenoid hydrocarbons, when Clar number increases the absorption bands shift to shorter wavelength, and the stability of these isomers also increases. Motivated by Clar's aromatic sextet theory, three types of polynomials (sextet polynomial, Clar polynomial, and Clar covering polynomial) were defined, and Randić's conjugated circuit model was also established. In this survey we attempt to review some advances on Clar's aromatic sextet theory and Randic's conjugated circuit model in the past two decades. New applications of these polynomials to fullerenes, and calculation methods of linear independent and minimal conjugated circuit polynomials of benzenoid hydrocarbons are also presented.
\end{abstract}

Keywords: Sextet polynomial, Clar polynomial, Clar covering polynomial, Linearly independent and minimal conjugated circuit polynomial, $k$-Resonance, Benzenoid hydrocarbon, Fullerene.

\section{INTRODUCTION}

The aromaticity reflects extra stability of certain types of conjugated systems due to the nature of molecular orbitals. The resonance energy, calculated from experimental measurements, denotes the energy gain or loss due to the interaction between Kekule structures, and represents the extra stability of the conjugated system. There have been distinct approaches developed to estimate the resonance energy. To deal with the problem in semiempirical valencebond view, different VB based models (see [1] for details) were built successively and hierarchically, following Pauling and Wheland [2]. Among these models, Clar's aromatic sextet theory is mainly based on experimental observations, which describes the aromaticity of benzenoid hydrocarbons. In this survey we attempt to review some advances on Clar's aromatic sextet theory and Randic's conjugated circuit model for polyhexes (i.e., benzenoid hydrocarbons (benzenoid systems)), and coronoid hydrocarbons (coronoid systems), as well as fullerenes.

We first discuss three polynomials on the aromaticity of polyhexes. Then we extend the discussions to investigate the stability of fullerenes.

The sources of Clar's aromatic sextet theory seem to be the paper of chemists Armit and Robinson [3] and the work of physical chemist Hückel [4-6]. The important role of 6membered conjugated cycles among $4 k+2$ conjugated cycles may also have inspired Clar's aromatic sextet theory, sextet polynomial, Clar polynomial, and Clar cover polynomial. In Clar's aromatic sextet theory, delocalized

*Address correspondence to this author at the School of Mathematical Sciences, Xiamen University, Xiamen, Fujian 361005, China; Tel: 865922182832; Fax: 865922580752; E-mail: fjzhang@xmu.edu.cn electrons in a 6-conjugated cycle are denoted by a circle, and as stated in Gutman [7] a Clar structure (Clar formula) consisting of circles satisfies the following three rules:

(a) Circles are never drawn in adjacent hexagons,

(b) The remainder of the polyhex obtained by the deletion of the vertices of the hexagons that possess circles must be empty or have a Kekulé structure, and

(c) As many circles as possible are drawn subject to the constraints (a) and (b).

If we draw some circles with only the constraints (a) and (b), we obtain a generalized Clar structure (or sextet pattern).

Clar observed that for isomeric benzenoid hydrocarbons when the number of the circles of Clar structures (called Clar number) increases, the absorption bands shift to shorter wavelength, and the stability of the isomers also increases. In his book [8], Clar provided many examples to support his observation and built Clar's aromatic sextet theory. Recently, topgraphical features of the molecular electrostatic potential of a series of polycyclic aromatic benzenoid hydrocarbons have been analyzed at B3LYP/6-31+G(d,p) and MP2/6$31+\mathrm{G}(\mathrm{d}, \mathrm{p})$ levels. The theoretical results fully support Clar's aromatic sextet theory [9].

In the study of Clar's aromatic sextet theory, the first task is to determine the Clar number.

For small benzenoid hydrocarbons we can easily find a Clar structure by pen and paper. The Clar structures of some large benzenoid hydrocarbons are exemplified in Fig. (1). For these examples, the conditions (a) and (b) can be easily verified, while the condition (c) on the maximality of the number of circles was proved in [10]. For more examples and further discussions, the reader is referred to [11-16]. 

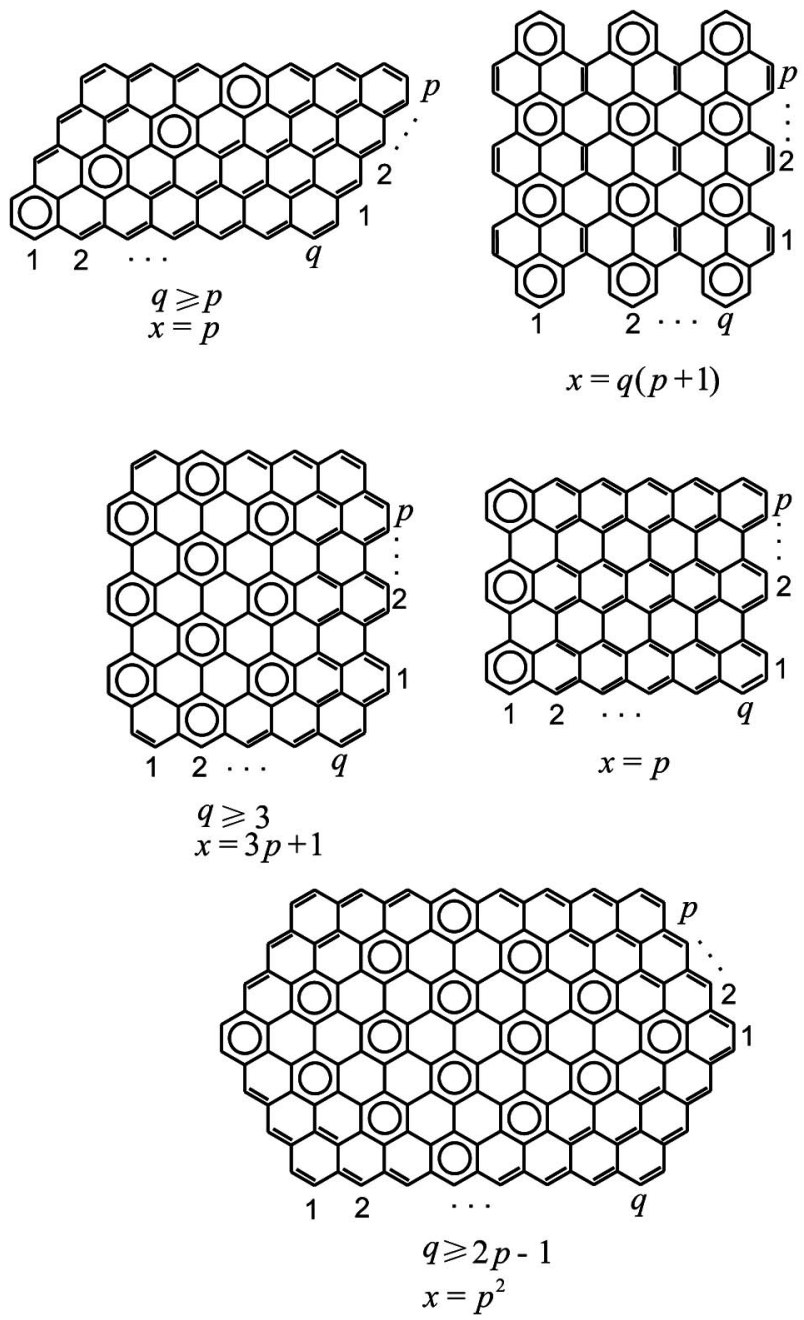

Fig. (1). Clar formulas of some classes of benzenoid systems, possessing $x$ aromatic sextets.

Hansen and Zheng $[17,18]$ computed the Clar number of a benzenoid hydrocarbon by the integer linear programming and conjectured that the linear programming relaxation was sufficient for the general case. The conjecture has been proved by Abeledo and Atkinson in [19].

A further problem is whether we can refine the idea of Clar number. A reasonable assumption is that all generalized Clar structures contribute to the resonance energy. From this point of view, Hosoya and Yamaguchi defined the sextet polynomial [20]. Another reasonable assumption is that the maximal generalized Clar structures (which are not a proper subset of another generalized Clar structure) contribute the most to the resonance energy. From this point of view, ElBasil and Randi c' defined the Clar polynomial [21]. Inspired by Clar structure, we reasonably thought that, for the resonance energy of a benzenoid hydrocarbon, besides the aromatic 6-circuits all double bounds should also be considered. This motivated us to define the Clar cover of a benzenoid hydrocarbon and the counting polynomial, Clar covering polynomial [22].
The conjugated circuit model, a resonance-theoretic model, was introduced by Randic' [23-25] in 1976 for the study of aromaticity and conjugation in polycyclic conjugated systems. It considers contributions of not only 6 -membered rings but also all $(4 k+2)$-membered conjugated circuits, as well as negative contributions of $4 k$ membered conjugated circuits to the resonance energy. The model was motivated from an empirical point of view elaborating the Clar aromatic sextet theory [8]. The conjugated-circuit model has also a firm quantum mechanical basis $[1,26,27]$. It can be derived rigorously from the Pauling-Wheland resonance theory [28-31] via a Simpson-Herndon model Hamiltonian [32-34]. The conjugated circuit model can be applied to more general cases. For example, Manoharana et al. [35] investigated the stability of fullerenes predicted by the topological resonance energy (TRE) model and the conjugated circuit model, and Babi $c^{\prime}$ and Trinajsti $c^{\prime}$ [36] reported the resonance energies (REs) of several fullerenes with 4-membered rings and their isomers with only 5- and 6-membered rings, using the conjugated-circuit model and the TRE model.

Here we consider only the conjugated circuit model for benzenoid hydrocarbons. The linear independent and minimal conjugated circuit polynomials ( LMCC polynomials) are defined explicitly, and a recursive method and analytical expressions for calculation of LMCC polynomials are discussed.

Finally we mention some results on $k$-resonance ( $k$ cycle resonance) in polyhexes, open end nanotubes, toroidal polyhexes, Klein-bottle polyhexes, and fullerenes as well.

\section{SEXTET POLYNOMIAL}

Let $G$ denote a benzenoid system. A generalized Clar structure ( sextet pattern) of $G$ is a set of disjoint hexagons of $G$, in each hexagon of which a circle is drawn, such that the deletion of the vertices of such hexagons together with their incident edges results in a graph with a perfect matching or an empty graph. To count sextet patterns of a benzenoid hydrocarbon $G$, Hosoya and Yamaguchi [20] defined the sextet polynomial $B_{G}(x)$ as follows.

$B_{G}(x)=\sum_{i=0}^{C(G)} r(G, i) x^{i}$

where $r(G, i)$ is the number of sextet patterns of $G$ with $i$ hexagons (or generalized Clar structures with $i$ cycles), and $C(G)$ is Clar number, the maximum size of sextet patterns. The concepts of sextet pattern and Clar number have been naturally extended to polycyclic conjugated hydrocarbons, such as coronoid systems, carbon nanotubes, and fullerenes, etc.

The sextet polynomial has some interesting mathematical properties. Hosoya and Yamaguchi [20], and Ohkami and Hosoya [37] found that there is a one-to-one correspondence between the sextet patterns and the Kekule structures for a catacondensed benzenoid hydrocarbon $G$, i.e. 
$B_{G}(1)=K(G)$,

where $K(G)$ denotes the number of Kekulé structures of $G$. They conjectured that for any benzenoid hydrocarbon (polyhex graph) which has at least one Kekulé pattern, there exists a one-to-one correspondence between Kekulé structures and sextet patterns. Two proofs of the conjecture were given by $\mathrm{He}$ and $\mathrm{He}$ [38], and by Ohkami [39]. But the proofs are not complete and have some errors. Zhang and Guo [40, 41] gave an explicit definition of super sextets of generalized polyhexes and a new proof of the OhkamiHosoya conjecture.

Zhang and Chen [42] showed that each hexagon of a benzenoid system $B$ forms a sextet pattern, i.e., $r(B, 1)$ is equal to the number of hexagons of $B$, if and only if $B$ is normal. The similar results hold for normal coronoid systems [43] and plane elementary bipartite graphs [44].

The sextet polynomial can be formally differentiated with respect to $x$ as $B_{G}^{\prime}(x)=\frac{d}{d x} B_{G}(x)$, where the derivative can be expressed as the sum of sextet polynomials of some subgraphs of $G$.

Theorem 2.1 [45] Let $G$ be a benzenoid system. Then

$B_{G}^{\prime}(x)=\sum_{h} B_{G-h}(x)$,

where the summation goes over all hexagons $h$ of $G$ and $G-h$ denotes the subgraph obtained from $G$ by deleting hexagon $h$ with incident edges and vertices.

Randi c ${ }^{\prime}$ [46] pointed out that the quotient $\frac{B_{G}^{\prime}(1)}{B_{G}(1)}$ can be regarded as a measure of the total aromaticity of a benzenoid $G$. In the following subsections we introduce some mathematical properties and chemical applications of sextet polynomials in various chemical graphs.

\subsection{Benzenoid Chains and Cyclo-Polyphenacenes}

How to compute the sextet polynomial for a benzenoid hydrocarbon? This is a problem of importance. It is well known that there are recurrence relations for computing almost all polynomials with applications in chemistry such as the characteristic polynomial, the independent polynomial and the matching polynomial of a graph. In [47], Gutman et al. gave a method of recurrence to compute the sextet polynomials for cata-condensed benzenoids. Gutman [48] found that for any benzenoid chain (unbranched catacondensed benzenoid hydrocarbon) there is a bijection between its generalized Clar structures and $k$-matchings of the corresponding Gutman (caterpillar) tree.

Recall that in graph theory, a caterpillar tree is a tree in which the removal of all its pendant vertices (vertices of degree 1) results in a path. In other words, let $v_{1}, v_{2}, \cdots$ be a path. If we join each of $n_{i}$ vertices to a vertex $v_{i}$ by an edge, $i=1,2, \cdots$, then a caterpillar tree is obtained.
For a benzenoid chain $B$, its corresponding Gutman tree is defined by the following construction: For the kink hexagons and the end hexagons of $\mathrm{B}$, represent each of them by an edge. Then join these edges successively to obtain a path. If $B$ contains $n_{i}$ (linear annulated) hexagons between the hexagons corresponding to the successive edges $v_{i-1} v_{i}$ and $v_{i} v_{i+1}$, then we add $n_{i}$ new vertices and join each of them to $v_{i}$ by an edge (see Fig. 2 for an example). Gutman [48] and El-Basil [49] proved the following result.

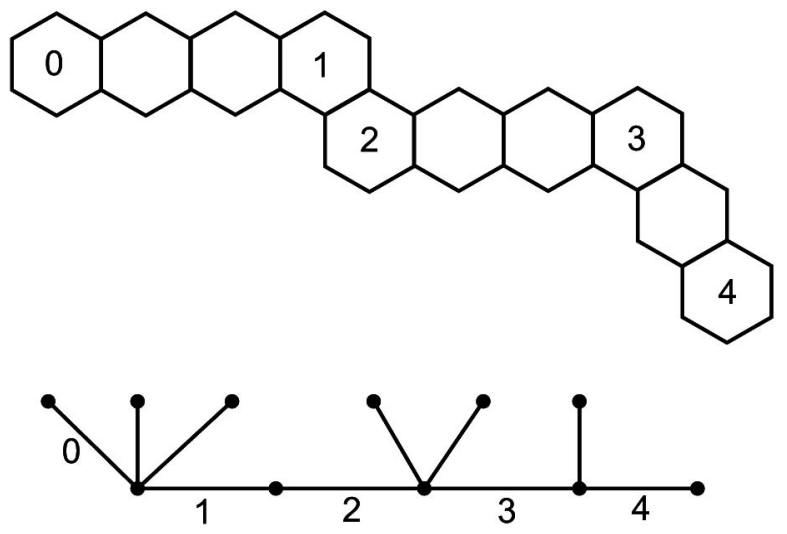

Fig. (2). A benzenoid chain and its corresponding Gutman tree.

Theorem 2.2 [48, 49] Let $B$ be a benzenoid chain and $G$ its corresponding Gutman tree. Then $r(B, k)=m(G, k)$, where $r(B, k)$ is the number of sextet patterns of $B$ with $k$ hexagons and $m(G, k)$ is the number of $k$-matchings of $G$.

Considering the contribution of Clar structure to the resonance energy, we introduce a quasi-order on benzenoid hydrocarbon isomers to compare their resonance energy. For two benzenoid isomers $B_{1}$ and $B_{2}$, if $r\left(B_{1}, k\right) \geq r\left(B_{2}, k\right)$ for $k=0,1,2, \cdots$, then we say $B_{1}$ is $s$-greater than $B_{2}$ and write $B_{1}>B_{2}$. If both $B_{1}>B_{2}$ and $B_{2}>B_{1}$ hold, then $B_{1}$ and $B_{2}$ are said to be $s$-equivalent. If neither $B_{1}>B_{2}$ nor $B_{1}<B_{2}$ holds, then $B_{1}$ and $B_{2}$ are incomparable. Clearly, two $s$ equivalent benzenoid chains may have the same sextet polynomial but need not be isomorphic. Based on the number of $k$-matchings of a graph, we can define a similar quasi-order ( $m$-greater) for graphs with the same number of vertices. If for two graphs $G_{1}$ and $G_{2}$, $m\left(G_{1}, k\right) \geq m\left(G_{2}, k\right), k=0,1,2, \cdots$, then we say $G_{1}$ is $m$ greater than $G_{2}$ and write $G_{1} \succ G_{2}$ [50]. By Theorem 2.2 we have:

Theorem 2.3 [51] Let $B_{1}$ and $B_{2}$ be two benzenoid chains with the same number of hexagons and $G_{1}$ and $G_{2}$ are the Gutman trees of $B_{1}$ and $B_{2}$, respectively. Then $B_{1}>B_{2}$ if and only if $G_{1} \succ G_{2}$.

Using Theorems 2.2 and 2.3 we can determine the extreme benzenoid chains with respect to their Clar aromatic 
sextets. In [51], it is showed that the minimal benzenoid chain is a linear chain, and the last four minimal benzenoid chains are also determined. On the other hand, the maximal benzenoid chains are $k^{*}$-cycle resonant benzenoid chains (the benzenoid chains whose hexagons are all kinks except the first and last one) and the second ones are the benzenoid chains whose hexagons are all kinks except the first and last two. Note that [51] contains an error in describing the second maximal benzenoid chains.

Similar to [51] concerning the ordering for benzenoid chains, the authors also consider the ordering of cyclopolyphenacenes (including the special case of primitive coronoids). The cyclo-polyphenacenes can be obtained from a chain of hexagons by identifying one edge of an end hexagon with an edge of the other end hexagon so that each hexagon is adjacent to exactly two hexagons. We can compute the sextet polynomial of cyclo-polyphenacenes recurrently with the help of matching polynomial. For isomeric cyclo-polyphenacenes, we introduce a quasi-order to compare their resonance energy. For this aim, we need to define the generalized crown corresponding to a cyclopolyphenacene. Recall that a generalized crown is a graph in which the removal of all its end vertices (vertices of degree 1) results in a cycle. In other words, let $v_{1} v_{2} \cdots v_{n} v_{1}$ be a cycle. If we join each of $m_{i}$ new vertices by an edge to the vertices $v_{i}$, for $i=1,2, \cdots, n$, then a generalized crown is obtained. For a cyclo-polyphenacene $B$, we can define a corresponding generalized crown (as illustrated in Fig. 3) and prove the following theorem.

Theorem 2.4 [52] Let $B$ be a cyclo-polyphenacene and $G$ be its corresponding generalized crown. Then the number of sextet patterns of $B$ having precisely $i$ hexagons is equal to the number of $i$-matchings of $G$ for any non-negative integer $i$.

Theorem 2.4 was used to solve "Hosoya's mystery" [53, 54] concerning the coincidence between the characteristic polynomial of a cycle and the polynomial of Kekulé structure count of a prime coronoid. For details, the reader is referred to [55].

With the help of matching polynomial, Theorem 2.4 can be also used to compute sextet polynomials of cyclopolyphenacenes. Some examples were given in [52]. Similar to the case of benzenoid chains, the ordering of cyclopolyphenacenes can also be set up. Then we have

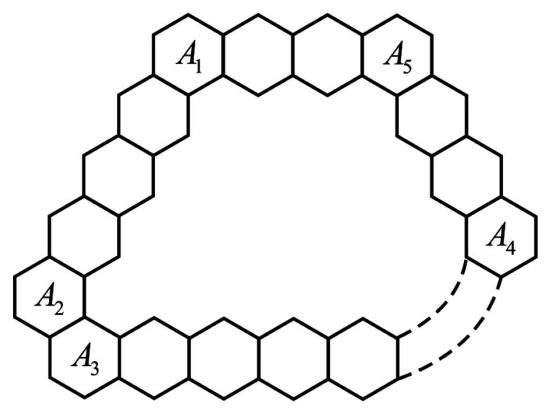

Theorem 2.5 [51] Let $B_{1}$ and $B_{2}$ be two cyclopolyphenacenes with the same number of hexagons, with $G_{1}$ and $G_{2}$ their corresponding generalized crowns, respectively. Then $G_{1} \succ G_{2}$ (strictly) if and only if $B_{1}>B_{2}$ (strictly) .

Theorem 2.4 reduces the ordering problem of cyclopolyphenacenes (with fixed number of Clar structures) to the ordering problem of the number of $i$-matchings of general crowns. Using Theorems 2.4 and 2.5, and some old results in [50], the authors of [51] determined the minimal, second minimal to seventh minimal cyclo-polyphenacenes with respect to the number of Clar's sextets. They also determined the maximal and second maximal family of cyclopolyphenacenes with respect to their number of Clar structures. For details, the reader is referred to [51].

\subsection{Resonant Patterns and Kekulé Structures - Alternant Case}

The one-to-one correspondence between the sextet patterns and Kekulé structures was first revealed for thin benzenoids by Hosoya and Yamaguchi as follows.

Theorem 2.6 [20] For a catacondensed benzenoid system $H, B_{H}(1)=K(H)$.

For the coronene $G$ (see Fig. 4), its sextet polynomial $B_{G}(x)=1+7 x+9 x^{2}+2 x^{3}$. So $B_{G}(1)=19<K(G)=20$. In fact the coronene is the critical forbidden subgraph for the above relation (2). This can be expressed in the following theorem obtained by Zhang and Chen [56], which was reproved later in a novel approach [57]. A subgraph $H$ of a graph $G$ is called nice if $G-V(H)$ either has a perfect matching or is empty.

Theorem 2.7 [56] For a hexagonal system $H$ with perfect matchings, $B_{H}(1) \leq K(H)$, and equality holds if and only if $\mathrm{H}$ contains no coronene as its nice subgraph.

By introducing super rings in sextet patterns of a benzenoid system, a general one-to-one correspondence between sextet patterns and Kekulé patterns can be established (see [40, 41]). For example, the exterior boundary of coronene as a super ring is added to the central hexagon to produce a new sextet pattern.

For the general alternant case -- plane bipartite graphs $G$ with perfect matchings, Gutman [58] and John [59]

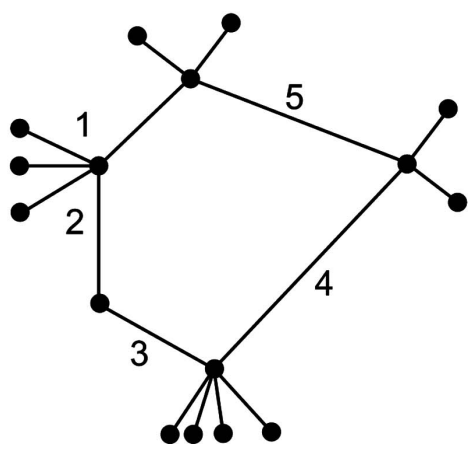

Fig. (3). A cyclo-polyphenacene and its corresponding generalized crown. 
independently defined resonant polynomials and cell polynomials to be count polynomials of resonant patterns. They extend sextet polynomials of benzenoid systems, by replacing hexagons of a sextet pattern with even inner faces. Let $r(G)$ denote the number of resonant patterns of $G$. John et al. [60] obtained Theorem 2.8; and a refined result -Theorem 2.9 was obtained in $[61,62]$.

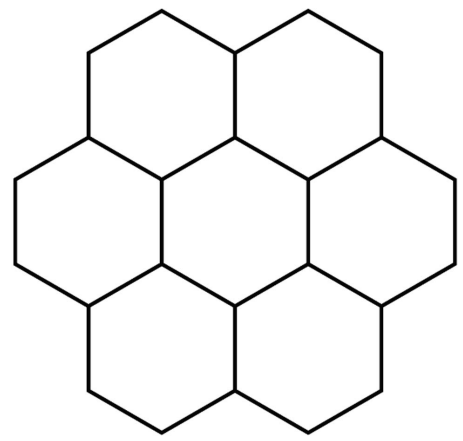

Fig. (4). Coronene.

Theorem 2.8 [60] For a plane bipartite graph $G$, $r(G) \leq K(G)$.

Theorem 2.9 [62, Theorem 3.2.1] Let $G$ be a 2connected plane bipartite graph with perfect matchings. Then $r(G) \leq K(G)$, and equality holds if and only if there do not exist disjoint cycles $\mathrm{R}$ and $\mathrm{C}$ such that (a) $R$ is a facial boundary lying in the interior of $C$ and (b) $C \cup R$ is a nice subgraph of $G$.

\subsection{Resonant Patterns and Kekulé Structures - Non- Alternant Case}

From the above subsection, we see that the correspondence between resonant patterns and Kekulé patterns relies strongly on the existence of the root perfect matching of a plane bipartite graph [63, 64]. This is not suitable for the non-alternant case (non-bipartite plane graphs).

By applying a novel approach, mathematical induction and the principle of inclusion and exclusion in combinatorics, Zhang and He [65] showed that for any plane graphs, the number of perfect matchings is not less than the number of resonant patterns. This generalizes the corresponding results in benzenoid systems and plane bipartite graphs. Applications to fullerenes (planar cubic graphs with only pentagonal and hexagonal faces) were also discussed.

For example, the resonant patterns of corannulene (Fig. 5) are as follows:

$\varnothing,\left\{C_{1}\right\},\left\{C_{2}\right\}$ $\left\{C_{3}\right\},\left\{C_{4}\right\},\left\{C_{5}\right\},\left\{C_{1}, C_{3}\right\},\left\{C_{1}, C_{4}\right\},\left\{C_{2}, C_{4}\right\},\left\{C_{2}, C_{5}\right\},\left\{C_{3}, C_{5}\right\}$.

Hence

$$
\begin{aligned}
& B_{\text {corannulene }}(x)=1+5 x+5 x^{2} . \text { It is computed [66] that } \\
& K(\text { corannulene })=11 . \\
& r(\text { corannulene })=K(\text { corannulene })=11 .
\end{aligned}
$$
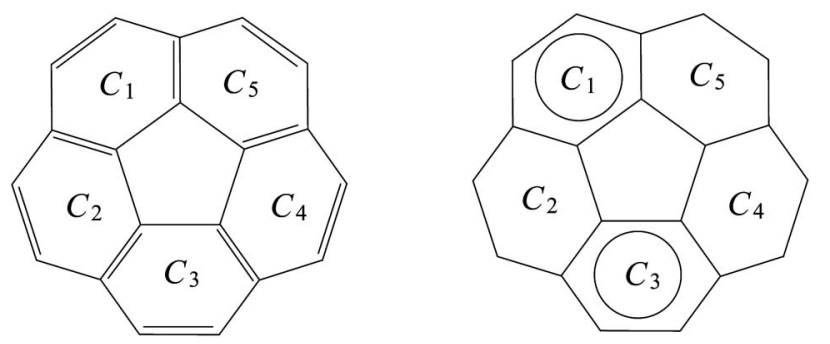

Fig. (5). Corannulene.

For the well-known fullerene--icosahedron $\mathrm{C}_{60}$ (Fig. 6 (left)), El-Basil [67] first found that its Clar number equals 8 . Since it has the Fries structure so that each hexagon is alternating, any set of disjoint hexagons always forms a sextet pattern. Based upon this, Shiu et al. [68] computed the sextet polynomial of $\mathrm{C}_{60}$ as

$$
B_{\mathrm{C}_{60}}(x)=5 x^{8}+320 x^{7}+1240 x^{6}+1912 x^{5}+1510 x^{4}+660 x^{3}+160 x^{2}+20 x+1 .(8)
$$

Ye et al. [69] showed that every hexagon of a fullerene is resonant, determined all the other eight 3-resonant fullerenes (i.e. every set of at most three disjoint hexagons forms a sextet pattern) and proved that any independent hexagons of a 3-resonant fullerene graph form a sextet pattern. So the sextet polynomials of the other eight 3 -resonant fullerene graphs are computed by counting sets of disjoint hexagonal faces (see [69] for details).

For such 3-resonant fullerene graphs $F$, we can confirm that the $B_{F}(1)<K(F)$. In general, Zhang and He showed the following result.

Theorem 2.10 [65] For any plane graph $G$, $r(G) \leq K(G)$.

For all fullerene graphs, Sereni and Stehlík [70] proved the following result, which was conjectured earlier by Zhang and $\mathrm{He}$ in [65].

Theorem 2.11 [70] For every fullerene graph $F$, $B_{F}(1)<K(F)$.

\subsection{Stability Indicators}

For benzenoid hydrocarbons, both the Clar number and Kekulé count can measure their stabilities. However, Austin et al. [71] constructed 20 distinct fullerene isomers of $\mathrm{C}_{60}$ whose Kekulé counts surpass the Kekulé count (12500) of icosahedral $\mathrm{C}_{60}$. So, the maximality of Kekulé counts of fullerene isomers may not correspond to the highest stability. Zhang et al. [72] turned to investigating a significant role of the Clar numbers of fullerenes in their stabilities. Zhang and Ye [73] obtained the sharp upper bound for the Clar number of fullerenes as follows.

Theorem 2.12 [73] Let $F_{n}$ be a fullerene with $n$ vertices. Then $c\left(F_{n}\right) \leq\left\lfloor\frac{n-12}{6}\right\rfloor$.

They also showed that there are infinite many fullerene graphs whose Clar number can achieve this upper bound, 

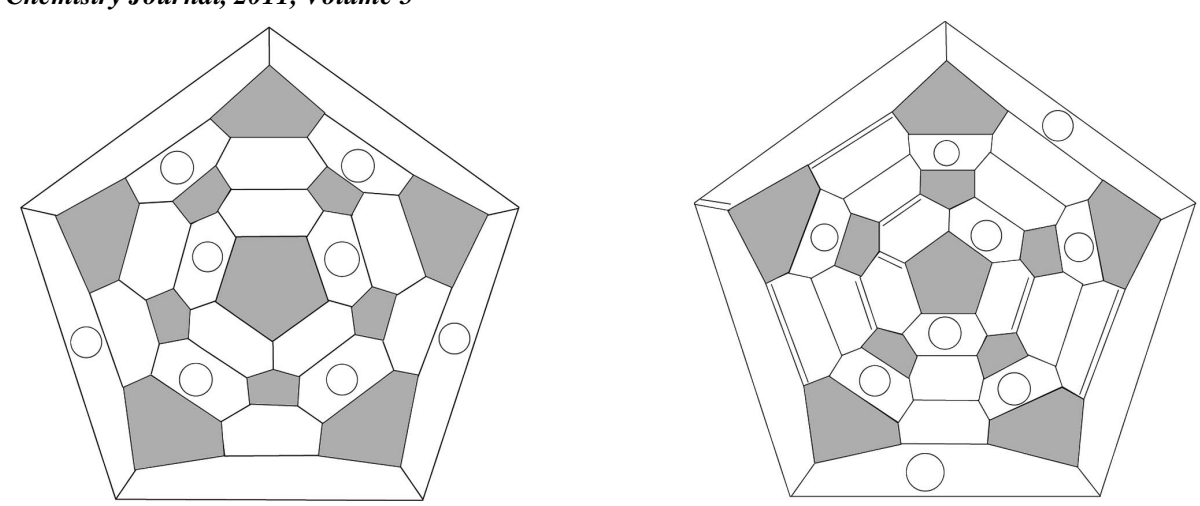

Fig. (6). Clar formulas of $\mathrm{C}_{60}(60: 1812)$ (left) and $\mathrm{C}_{70}$ (70:8149) (right).

including $\mathrm{C}_{60}$ and $\mathrm{C}_{70}$, and zigzag and armchair carbon nanotubes as well; Theorem 2.12 shows that none of fullerene graphs are "all-benzenoids" [74, 75]. Combining theorem 2.12 and some construction of Clar formula of fullerenes, they found that the experimentally characterized $\mathrm{C}_{60}, \mathrm{C}_{70}, \mathrm{C}_{76}, \mathrm{C}_{84}: 22 D_{2}$ (IV) and $\mathrm{C}_{84}: 23 D_{2 d}$ (II) attain the maximum Clar number among their fullerene isomers.

Ye and Zhang [76] have rigorously proved that exactly 18 fullerenes with 60 atoms (including the icosahedral $\mathrm{C}_{60}$ ) achieve the maximum Clar number 8 . A comparison shows that none of these 18 fullerenes belongs to the collection of the 20 fullerene isomers with Kekulé counts surpassing 12500 in [71]. That is, the Clar numbers of these 20 fullerene isomers are all less than 8. Hence, a combination of Clar number and Kekulé count as a stability predictor distinguishes uniquely the icosahedral $\mathrm{C}_{60}$ from its all 1812 fullerene isomers.

Furthermore, W. Sun and F. Wang in Lanzhou University have computed the sextet polynomials of all fullerene isomers of $\mathrm{C}_{60}$ and $\mathrm{C}_{70}$. It is known that $\mathrm{C}_{60}$ and $\mathrm{C}_{70}$ have 1812 and 8149 fullerene isomers, respectively [77]. From their computational results we can see that $C_{60}(60: 1812)$ is a unique fullerene isomer of $\mathrm{C}_{60}$ with the maximum number of sextet patterns 5828, and the isomer 60:1809 has the second maximum number of sextet patterns 3970. So $\mathrm{C}_{60}(60: 1812)$ has a much larger sextet pattern count than the isomer 60:1809. Similarly, the experimentally characterized 70:8149 is a unique fullerene isomer of $\mathrm{C}_{70}$ with the maximum sextet pattern count 18714 , and 70:7106 has the second maximum sextet pattern count 17463 . Such partial computational results are listed in Table $\mathbf{1}$.

How to compute Clar numbers of fullerenes is an interesting problem in both mathematics and theoretical chemistry. Up to this date, an effective general way has not been found for this problem. It is worthwhile to seek appropriate combinations of Clar numbers with other invariants as stability predictors of fullerenes.

\section{CLAR COVERING POLYNOMIALS}

The definition for a sextet pattern of a generalized benzenoid system $B$ was slightly modified in [22]. We add a Kekulé structure of $H-Q$ to a sextet pattern $Q$ to get a vertex-cover of $B$, and we call such a vertex-cover a Clar cover of a (generalized) benzenoid system $B$. In other words, a spanning subgraph $C$ of $B$ is said to be a Clar cover of $B$ if each of its components is either a hexagon or an edge. Then the Clar covering polynomial of $B$ is defined as:

$$
\zeta(x)=\zeta(B, x)=\sum_{i=0}^{C(B)} z(B, i) x^{i},
$$

where $z(B, i)$ denotes the number of Clar covers of $B$ having precisely $i$ hexagons (refer to [22, 78-81]).

This polynomial was used to conveniently compare topological indices of some types of benzenoid isomers [80]. It is also called "Zhang-Zhang polynomial" in a series of papers due to Gutman et al. [82-88].

Table 1. The First, Second and Third Maximum $B_{F}(1)$ Fullerene Isomers of $C_{60}$ and $C_{70}$

\begin{tabular}{|c|c|c|}
\hline Isomers $\boldsymbol{F}$ & Sextet polynomial $\boldsymbol{B}_{F}(\boldsymbol{x})$ & $\boldsymbol{B}_{\boldsymbol{F}}(\mathbf{1})$ \\
\hline \hline $60: 1804$ & $x 8+48 x 7+377 x 6+934 x 5+1061 x 4+588 x 3+157 x 2+20 x+1$ & 3187 \\
\hline $60: 1809$ & $x^{8}+98 x^{7}+594 x^{6}+1250 x^{5}+1232 x^{4}+616 x^{3}+158 x^{2}+20 x+1$ & 3970 \\
\hline $60: 1812$ & $5 x^{8}+320 x^{7}+1240 x^{6}+1912 x^{5}+1510 x^{4}+660 x^{3}+160 x^{2}+20 x+1$ & 17289 \\
\hline $70: 7716$ & $3 x 9+303 x 8+1871 x 7+4478 x 6+5435 x 5+3613 x 4+1307 x 3+253 x 2+25 x+1$ & 17463 \\
\hline $70: 7106$ & $20 x^{9}+343 x^{8}+1902 x^{7}+4500 x^{6}+5474 x^{5}+3634 x^{4}+1311 x^{3}+253 x^{2}+25 x+1$ & 18714 \\
\hline $70: 8149$ & $25 x^{9}+375 x^{8}+2065 x^{7}+4715 x^{6}+5958 x^{5}+3940 x^{4}+1355 x^{3}+255 x^{2}+25 x+1$ & \\
\hline
\end{tabular}




\subsection{Basic Properties}

We first introduce some basic properties of Clar covering polynomial of a generalized benzenoid system $B$.

Theorem 3.1 [22] Let $B$ be a benzenoid system. Then we have the following properties for the Clar covering polynomial of $B$ :

1. $\zeta(B, 0)=K(B)$,

2. the degree of the polynomial $\zeta(B, x)$ is $C(B)$, the Clar number of $B$,

3. the coefficient of the highest degree term, $z(B, C(B))$ equals the number of Clar formulas of $B$,

4. $z(B, 1)=h_{1}(B)$, the first Herndon number.

The Clar covering polynomial closely relates to the sextet polynomial via a transformation of polynomials.<smiles>c1ccccc1</smiles><smiles>c1ccccc1</smiles>

\section{proper sextet}

\section{improper sextet}

Fig. (7). Modes of proper sextet and improper sextet.

Let $B$ be a benzenoid system with a perfect matching (Kekulé structure or 1-factor) $M$. A conjugated (or alternating) hexagon of $B$ is called a proper sextet if the extreme right vertical edge belongs to $M$; an improper sextet otherwise, as illustrated in Fig. (7). Use Let $a(B, i)$ denote the number of perfect matchings of $B$ which contains precisely $i$ proper sextets for $0 \leq i \leq C(B)$. Then we have

$\sum_{i=0}^{C(B)} a(B, i)=K(B), a(B, i)>0$ for all $0 \leq i \leq C(B)$, and $a(B, 0)=1$. Further, these $a(B, i)$ become the coefficients of a new polynomial expression for the Clar covering polynomial in variable $(x+1)$.

Theorem 3.2 [79] Let $B$ be a benzenoid system with a perfect matching. Then the Clar covering polynomial $\zeta(B, x)$ of $B$ can be expressed in the following form:

$\zeta(B, x)=\sum_{i=0}^{C(B)} z(B, i) x^{i}=\sum_{i=0}^{C(B)} a(B, i)(x+1)^{i}$.

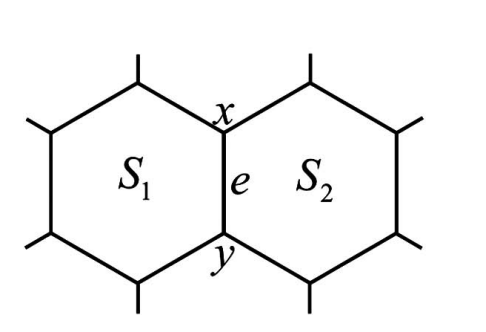

Via such a transformation, we can establish a relation with the sextet polynomial as follows.

Theorem 3.3 [79] Let $B$ be a benzenoid system with a perfect matching. For all $0 \leq i \leq C(B), a(B, i) \geq r(B, i)$ and all the equalities hold if and only if $B$ has no coronene $C$ (see Fig. 4) as its nice subgraph.

Corollary 3.4 [79] Let $B$ be a benzenoid system with a perfect matching. Then

$\sum_{i=0}^{C(B)} a(B, i) x^{i}$ is the sextet polynomial of $B$ if and only if $B$ has no coronene as its nice subgraph.

For other interesting properties, the reader is referred to [79].

In general, the polynomial $\sum_{i=0}^{C(B)} a(B, i) x^{i}$ can be viewed as the revised sextet polynomial of $B$ which counts sextet patterns with super rings.

\subsection{Computation Approach}

Compared with the sextet polynomial, the Clar covering polynomial of a benzenoid system has one advantage in computation: it has general recurrence relations. This enables one to compute some significant topological indices of benzenoid systems as mentioned in Theorem 3.1 by some recurrence procedures.

Theorem 3.5 [22] Let $B$ be a generalized benzenoid system with the components $B_{1}, B_{2}, \ldots, B_{k}$. Then

$\zeta(B, x)=\prod_{i=1}^{k} \zeta\left(B_{i}, x\right)$.

Theorem 3.6 [22] Let $B$ be a generalized benzenoid system. Let $s_{1}$ and $s_{2}$ be two hexagons of $B$ having a common edge $e=x y$ (see Fig. 8 (left)). Then

$\zeta(B)=w \sum_{i=1}^{2} \zeta\left(B-s_{i}\right)+\zeta(B-x y)+\zeta(B-x-y)$,

where $B-s_{i}$ denotes the subgraph obtained from $B$ by deleting all vertices of $s_{i}$ together with incident edges.

Theorem 3.7 [22] Let $B$ be a generalized benzenoid system. Let $x y$ be an edge of a hexagon $s$ of $B$ which lies on the periphery of $B$ (see Fig. 8 (middle)). Then

$$
\zeta(B)=w \zeta(B-s)+\zeta(B-x-y)+\zeta(B-x y) \text {. }
$$
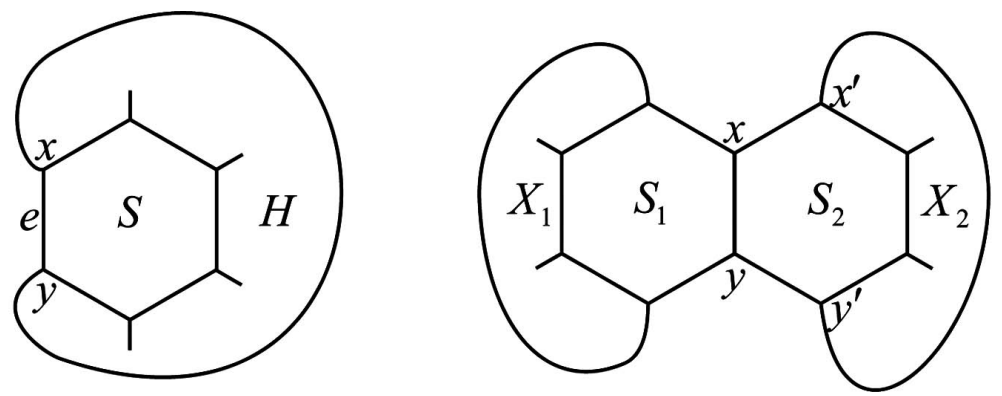

Fig. (8). Modes of hexagons $s_{1}$ and $s_{2}$ in a benzenoid system for some reduced procedures. 
Theorem 3.8 [22] Let $X_{1}$ and $X_{2}$ be two Kekuléan benzenoid systems which contain hexagons $s_{1}$ and $s_{2}$, respectively, as indicated in Fig. 8 (right) (or one of them be $\left.K_{2}\right)$. Let $X_{1}: X_{2}$ be a benzenoid system obtained by gluing $X_{1}$ and $X_{2}$ only along an edge $x y$ of $s_{1}$ and $s_{2}$. Then the Clar covering polynomial of $X_{1}: X_{2}$ is

$\zeta\left(X_{1}: X_{2}\right)=\zeta\left(X_{1}\right) \zeta\left(X_{2^{\prime}}\right)+\zeta\left(X_{1^{\prime}}\right) \zeta\left(X_{2}\right)-\zeta\left(X_{1^{\prime}}\right) \zeta\left(X_{2^{\prime}}\right)$

where $X_{i}=X_{i}-x-y$ for $i=1$ or 2 .

Corollary 3.4 and Theorem 3.8 can be used to derive a recurrence relation for sextet polynomial of cata-condensed benzenoid systems as follows.

Corollary 3.9 [62, Corollary 5.2.11] Let $X_{2}$ be a catacondensed benzenoid system. Then

$B_{L_{m}: X_{2}}(x)=m x B_{X_{2^{\prime}}}(x)+B_{X_{2}}(x)$,

where $L_{m}$ denotes the linear benzenoid chain of $m$ hexagons.

Clar covering polynomials have been computed for of some types of benzenoid and coronoid systems, such as benzenoid chains [22], parallelogram [62], multiple linear hexagonal chains [88], cyclo-polyphenacenes [89], and so on $[90,91]$.

\subsection{Applications to Resonance Energy}

Each of the quantities mentioned in points (1)-(4) of Theorem 3.1 was shown to relate to some kinds of the resonance energy. It is reasonable to expect that the Clar covering polynomial will also be somehow connected with the resonance energy.

Zhang et al. [78] established an approximation model of DRE with Clar covering polynomial of benzenoid hydrocarbons:

$R E=\alpha \sum_{i=0}^{C(B)} z(B, i) \varepsilon_{i}+\beta$.

For condensed aromatic hydrocarbons with Clar number $\leq 4$, the parameters $\alpha$ and $\beta$ and weights $\varepsilon_{i}(1 \leq i \leq 4)$ in the above Eq. (5), we determined $\varepsilon_{1}=1$, $\varepsilon_{2}=0.18, \varepsilon_{3}=0.14 \quad$ and $\varepsilon_{4}=27.33, \quad \alpha=1.940 \quad$ and $\beta=0.0113$. The correlation coefficient is 0.9971 and the mean error is 0.014 .

Gutman et al. [82] found good linear correlations between TRE and $\ln \zeta(x)$ for fixed values of $x$ lying in the interval $[0,2]$ as,

$R E \approx a \ln \zeta(x)+b$,

where $a$ and $b$ are constants. The special case of the above approximation (6) for $x=0$ is the usual approximation of resonance energy via Kekulé count:

$R E=a_{0} \ln K$.

In fact, the correlation coefficient $\mathrm{R}$ attains a maximum, and the average relative error attains a minimum, for some $x$ that considerably differs from zero. The unexpected finding is that the optimal value of $x$ is always remarkably close to unity. There is no statistically significant difference between the accuracy of the approximation for optimal $x$ and for $x=1$. This leads to the conclusion that $\zeta(1)$ is a quantity of some importance in the Clar theory of benzenoid molecules.

Further studies of Gutman et al. [83, 86] revealed certain hitherto concealed properties of resonance energies of benzenoid molecules, and their dependence on Kekulé- and Clar-structure-based parameters.

\subsection{An Extension to Fullerenes}

Similarly to the sextet polynomial, the Clar covering polynomial can be naturally extended to fullerene graphs. We also checked its role in the stability of fullerenes.

W. Sun and F. Wang also computed the Clar covering polynomials of all fullerene isomers of $\mathrm{C}_{60}$ and $\mathrm{C}_{70}$. Their computation results show that $\mathrm{C}_{60}(60: 1812)$ achieves the maximum $\zeta(1)=250967$, and the isomer 60:1809 has the second maximum $\zeta(1)=158829$. These are consistent with their sextet pattern counts. For $\mathrm{C}_{70}$, fullerene isomers 70:8149, 70:7716 and 70:7106 have the first, second and third maximum $\zeta(1)$, which are 1305863, 1289527 and

Table 2. The First, Second and Third Maximum $\xi(1)$ Fullerene Isomers of $C_{60}$ and $C_{70}$

\begin{tabular}{|c|r|c|}
\hline Isomers & Clar covering polynomial $\xi(x)$ & $\xi(1)$ \\
\hline \hline $60: 288$ & $2 x^{7}+82 x^{6}+1082 x^{5}+7168 x^{4}+24956 x^{3}+46144 x^{2}+42862 x+15745$ & 138041 \\
\hline $60: 1809$ & $x^{8}+106 x^{7}+1308 x^{6}+6996 x^{5}+20972 x^{4}+38968 x^{3}+46156 x^{2}+33092 x+11230$ & 158829 \\
\hline $60: 1812$ & $5 x^{8}+360 x^{7}+3620 x^{6}+16352 x^{5}+42000 x^{4}+66900 x^{3}+67650 x^{2}+41580 x+12500$ & 250967 \\
\hline $70: 7106$ & $20 x^{9}+523 x^{8}+5474 x^{7}+30727 x^{6}+104690 x^{5}+230671 x^{4}+337496 x^{3}+324001 x^{2}+188633 x+51068$ & 1273303 \\
\hline $70: 7716$ & $3 x^{9}+335 x^{8}+4569 x^{7}+28288 x^{6}+101191 x^{5}+229711 x^{4}+342961 x^{3}+333691 x^{2}+195714 x+53064$ & 1289527 \\
\hline $70: 8149$ & $25 x^{9}+600 x^{8}+5965 x^{7}+31970 x^{6}+106065 x^{5}+233215 x^{4}+345490 x^{3}+335385 x^{2}+194980 x+52168$ & 1305863 \\
\hline
\end{tabular}


1273303, respectively (see Table 2). We can see that the fullerene isomers 60:1812 and 70:8149 retain the maximum values in both sextet pattern count and Clar cover count among their fullerene isomers. However, the isomers 70:7716 and 70:7106 have the near $\zeta(1)$ 's, and are just exchanged, compared in the ranking in terms of sextet pattern counts.

\section{CLAR POLYNOMIAL}

There are various extensions of Clar structures. Herndon and Hosoya [92] alleviated rule (c) in the Introduction to define an (extended) Clar structure for calculating resonance energies with more accuracy: Clar structure may contain less than $C$ cycles, but no benzenoid ring should have proper or improper sextets. El-Basil and Randi c' et al. [21, 93-95] described such an extension of Clar structures and gave various construction approaches. For some large benzenoid systems, however, there are different explanations.

Shiu et al. [68] clarified such an extended Clar structure by replacing (c) with (d): the set of circles is maximal, i.e. no new cycle can be drawn using (a) and (b).

By using Clar covers, a more precise graph-theoretical definition of Clar structures was presented. Let $G$ be a plane or spherical graph with a perfect matching. A Clar cover $C$ of $G$ is called a Clar structure if the set of hexagons is maximal (in the sense of set-inclusion) in all Clar covers of $G$, and $C$ is called a proper Clar structure if the number of hexagons reaches the maximum in all Clar covers of $G$.

The count polynomial of Clar structures, referred to as the Clar polynomial, was defined by El-Basil and Randi c' $[93,94,95]$ for a benzenoid system $G$ :

$\xi(G, x)=\sum_{i=0}^{C(G)} \rho(G, i) x^{i}$,

where $\rho(G, i)$ denotes the number of Clar structures of $G$ with $i$ circles.

Randi c' et al. [95] gave an approach to compute the Clar polynomial of large benzenoids, and obtained the following results:

Theorem 4.1 [95] Let $B$ be a Kekuléan benzenoid system. Then

$\frac{d}{d x} \xi(B, x)=\sum_{h} \xi(B-h, x)$,

where the summation goes over all hexagons $h$ of $B$.

Theorem 4.2 [95] Let $B$ be a Kekuléan benzenoid. Then

$\xi(B, x)=\sum_{h} \int \xi(B-h, x) d x$

with the integrating constant $c=0$.

Shiu et al. [68] computed the Clar polynomial of the icosahedral $\mathrm{C}_{60}$ as follows.

$\xi\left(\mathrm{C}_{60}, x\right)=5 x^{8}+280 x^{7}+10 x^{6}$. which corrects the error in [67].

We now discuss an index $c c(B)$ related to Clar polynomial of a benzenoid system $B$. Let $c c(B)$ denote the number of Clar covers without alternating hexagons. Then

$\xi(B, 1) \leq c c(B)$.

The sextet rotation, transforming all proper sextets of a Kekulé structure of a benzenoid system $B$ into improper sextets, results in a directed tree on the set of Kekulé structures of $B$ with one root, denoted by $R(B)$. Let $n l(B)$ denote the number of non-leaves in $R(B)$.

Theorem 4.3 [57] Let $B$ be a benzenoid system with a perfect matching. Then $c c(B)=n l(B)$.

Theorem 4.4 [57] If a benzenoid system $B$ has a perfect matching and contains no coronene as its nice subgraph, then $\xi(B, 1)=c c(B)$.

If all Clar covers without alternating hexagons in a benzenoid system are identical to their Clar structures, i.e. $\xi(B, 1)=c c(B)$, we can obtain the Clar polynomial by enumerating Clar covers without alternating hexagons. In fact, the converse of Theorem 4.4 does not hold. For example, $\xi($ coronene, 1$)=c c($ coronene $)$. So we can easily obtain the Clar polynomial of coronene as $\xi($ coronene, $x)=2 x^{3}+3 x^{2}+2 x$, which, of course, agrees with the earlier result of Ref. [94]. In fact many benzenoid systems $B$ that contain coronene as its nice subgraph with $\xi(B, 1)=c c(B)$, have been constructed. For more details, the reader is reffered to [57].

\section{LINEARLY INDEPENDENT AND MINIMAL CONJUGATED CIRCUIT POLYNOMIALS OF BENZENOID HYDROCARBONS}

\subsection{Introduction}

The conjugated circuit model is a resonance-theoretic model, which was introduced by Randi $c^{\prime}$ in 1976 for the study of aromaticity and conjugation in polycyclic conjugated systems. Enumeration of conjugated circuits led to expressions for the resonance energy of polycyclic conjugated hydrocarbons [96]. In recent years, various investigations on the conjugated-circuit model have been made [96-104], such as quantum-mechanical and computational aspects of the conjugated-circuit model, the selection of the optimum parameters of the conjugatedcircuit model, and comparison between the conjugatedcircuit model and several other models for computing the resonance energies of benzenoid hydrocarbons.

In [105], Guo and Randi c' gave a strict definition of linearly independent and minimal conjugated circuits and $L M$-conjugated circuit polynomials as follows.

Definition 5.1 [105]. A set $S$ of linearly independent and minimal conjugated circuits of a Kekulé structure $K_{i}$ of a benzenoid hydrocarbon $B$ consists of a maximum number of linearly independent circuits of $B$ in which every circuit 
is a conjugated circuit of $K_{i}$, and has the minimum length. Denote a circuit of size $4 n+2$ in $S$ by $R_{n}$, and the summation expression of $S$ by $R\left(K_{i}\right)=\sum_{R_{j} \in S} R_{j}=\sum_{n=1,2, \cdots} r_{n}\left(K_{i}\right) R_{n}$, where $r_{n}\left(K_{i}\right)$ is the number of the circuits of size $4 n+2$ in $S$. The summation expression of all sets of $L M$-conjugated circuits of all Kekulé structures of $B$ is denoted by $R(B)=R=\sum_{K_{i}} R\left(K_{i}\right)=\sum_{n=l, 2, \cdots} r_{n} R_{n}$, where $r_{n}=\sum_{K_{i}} r_{n}\left(K_{i}\right)$.

The summation expression $R(B)$ of $L M$-conjugated circuits of $B$ is called $L M$ C-expressions or the $L M$ conjugated circuit polynomial (simply the LMCCpolynomial) of $\mathrm{B}$, which is a polynomial of degree one with multi-variants and may also be denoted by a sequence of numbers $\left(r_{l}, r_{2}, r_{3}, \cdots, r_{n}, \cdots\right)$, called the $L M C C$-code of $B$.

The $L M$-conjugated circuit polynomials of benzenoid hydrocarbons play a central role in the conjugated-circuit model, because the resonance energy $R E(B)$ of a benzenoid hydrocarbon $B$ is simply equal to $R(B) / K(B)$. Here $K(B)$ is the number of Kekule structures of $B$. The $L M-$ conjugated circuit polynomials had been also applied to calculate generalized bond orders of polycyclic conjugated hydrocarbons [106]. Thus the calculation of the LM conjugated circuit polynomials of benzenoid hydrocarbons becomes a fundamental problem on the conjugated-circuit model.

However, for a general case, the enumeration of $L M$ conjugated circuits of benzenoid hydrocarbons requires to construct all Kekulé structures and then to find a set of $L M$ conjugated circuits for every Kekulé structure. When the size of a molecule increases, the number of Kekule structures increases fast, and hence enumerating $L M$-conjugated circuits by this method becomes tedious.

Guo and Randi c' [105] investigated the properties and the construction of minimal conjugated circuits of benzenoid hydrocarbons, and gave the necessary and sufficient condition for a set of conjugated circuits of a benzenoid hydrocarbon to be linearly independent and minimal. Furthermore, they established some recursive relations for calculating the $L M$-conjugated circuit polynomials of several classes of benzenoid hydrocarbons, so that the $L M C C$-polynomials of the several classes of benzenoid hydrocarbons can be directly obtained from the LMCC polynomials and the Kekule structure counts of their subgraphs. Guo and Randic' [107] extended the recursive formulae for calculating $L M C C$-polynomials for both catacondensed benzenoid hydrocarbons and some families of structurally related pericondensed benzenoid hydrocarbons. There are still some classes of benzenoid hydrocarbons whose $L M C C$-polynomials cannot be obtained by the above recursive method. For general cases, Guo, Randi c', and Klein [108] further gave an analytical expression for the count of $L M$-conjugated circuits of $B$ which is based on the counts of Kekulé structures of selected subgraphs of $B$.
By using the method, the LMCC-polynomials of any benzenoid hydrocarbon can be obtained.

A benzenoid hydrocarbon $(B H)$ is a 2-connected plane graph whose every interior face is bounded by a regular hexagon. A connected subgraph of a $B H$ is said to be a $B H$-fragment ( $B H F)$. A 2-connected $B H F$ is said to be a generalized $B H(G B H)$. Let $B$ be a $B H$ or $G B H$. A bond of $B$ is said to be a fixed bond if it appears always as a double bond in every Kekulé structure), or always as a single bond. If $B$ contains no fixed bond, then $B$ is said to be normal; otherwise $B$ is said to be essentially disconnected. A normal component $B_{i}$ of $B$ is a maximal subgraph of $B$ with no fixed bond (possibly, $B_{i}=B$, that is, $B$ is normal). All normal components of $B$ are denoted by $B^{*}$. The boundary of an interior face of a $B H$ or $B H$-fragment $B$ is called a ring of $B$.

Definition 5.2 [105]. Let $s$ be a ring (i.e. a hexagon) of a benzenoid hydrocarbon $B$, and $K_{i}$ a Kekulé structure of $B$. A conjugated circuit $C$ of $K_{i}$ (simply, a $K_{i}$-conjugated circuit $C$ ) is said to be a minimal conjugated circuit of the ring $s$ if the interior of $C$ contains the interior of $s$ and $C$ has the minimum length. We also say that a $K_{i}$-conjugated circuit $C$ of $B$ is minimal if there is a ring $s$ in $B$ such that $C$ is a minimal conjugated circuit of $s$ (see Fig. 9).

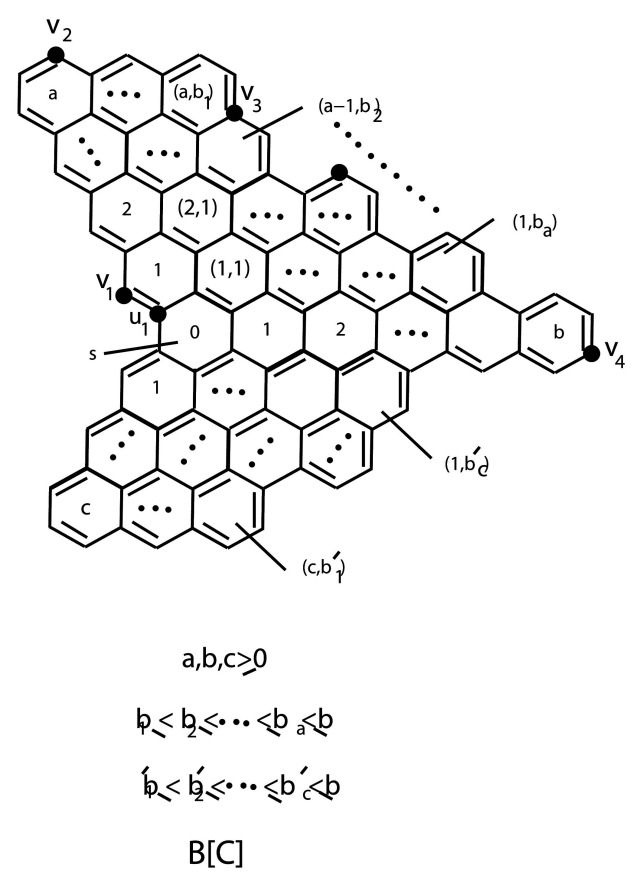

Fig. (9). Construction of minimal conjugated circuits of a ring $s$ of a benzenoid hydrocarbon $B$.

Theorem 5.1 [105]. Let $K_{i}$ be a Kekulé structure of a benzenoid hydrocarbon $B$, and let $C$ be a minimal conjugated circuit of a ring $s$ of $B$. Then $B[C]$ is one of 
the BHs shown in Fig. (9), and the $K_{i}$ double bonds in $B[C]$ are uniquely determined.

Theorem 5.2 [105]. Let $K_{i}$ be a Kekulé structure of a benzenoid hydrocarbon $B$. A set $S=\left\{C_{l}, C_{2}, C_{3}, \cdots, C_{t}\right\}$ of $K_{i}$-conjugated circuits of $B$ is a set of $L M$-conjugated circuits of $K_{i}$ if and only if for any ring $s_{j}$ in all normal components $B^{*}$ of $B$ there is exactly one circuit $C$ in $S$ such that $C_{j}$ is a minimal $K_{i}$-conjugated circuit of $s_{j}$.

\subsection{Recursive Method for Computing $L M$-Conjugated Circuit Polynomials of Benzenoid Hydrocarbons}

Theorem 5.2 establishes the theoretical basis of the partition of the $L M C$-expression of $B$ into the $L M C$ expressions of rings of $B$. The $L M C$-expression of a ring $s$ in $B$, denoted by $R_{s}(B)$, is determined by taking the summation expression of the minimal conjugated circuits of $s$, one for every Kekulé structure of $B$, and $R(B)=\sum_{s}^{B} R_{s}(B) . \quad R_{s}(B)$ may also be denoted by a sequence of numbers (ring code) $\left(r_{l}(s), r_{2}(s), \cdots, r_{n}(s), \cdots\right)$, where $r_{n}(s)$ is the coefficient of the term $R_{n}$ in $R_{s}(B)$. Theorem 5.2 also enables us to establish some recursive relations for enumeration of $L M$-conjugated circuits of $B$.

Definition 5.3 [105]. For an edge $e=u v$ of a benzenoid hydrocarbon $B$, let $B_{e}\left(B_{\bar{e}}\right)$ denote the labeled graph of $B$ for which the edge $e$ is labeled as double (single) bond, and Let $B_{e}^{*}\left(B_{\bar{e}}^{*}\right)$ denote the normal components of $B-u-v$ $(B-e) \quad\left[B_{e}^{*}\right.$ and $B_{\bar{e}}^{*}$ may be thought as the normal components of $B_{e}\left(B_{\bar{e}}\right)$, since $e$ is in fact a fixed double (single) bond in $\left.B_{e}\left(B_{\bar{e}}\right)\right]$. The subgraph of $B_{e}\left(B_{\bar{e}}\right)$ induced by the hexagons in $B_{e}\left(B_{\bar{e}}\right)$ which are not in $B_{e}^{*}$ $\left(B_{\bar{e}}^{*}\right)$ is denoted by $B_{e^{\prime}}\left(B_{\bar{e}}{ }^{\prime}\right)$. The contribution of all rings in $B_{e}^{*}\left(B_{\bar{e}}^{*}\right)$ to $R\left(B_{e}\right)\left(R\left(B_{\bar{e}}\right)\right)$ is denoted by $R^{*}\left(B_{e}\right)$
( $\left.R^{*}\left(B_{\bar{e}}\right)\right)$, and the contribution of all rings in $B_{e}{ }^{\prime}\left(B_{\bar{e}}{ }^{\prime}\right)$ to $R\left(B_{e}\right)\left(R\left(B_{\bar{e}}\right)\right)$ is denoted by $R^{\prime}\left(B_{e}\right)\left(R^{\prime}\left(B_{\bar{e}}\right)\right)$.

Clearly, $R\left(B_{e}\right)$ and $R\left(B_{\bar{e}}\right)$ are just the $L M C$ expressions of all the Kekulé structures of $B$ containing and not containing the edge $e$, respectively. Thus

$$
R(B)=R^{*}\left(B_{e}\right)+R^{*}\left(B_{\bar{e}}\right)+R^{\prime}\left(B_{e}\right)+R^{\prime}\left(B_{\bar{e}}\right)
$$

The above expressions give some partitions of $L M$ conjugated circuits of $B$, so that we can obtain $R(B)$ from its all parts. However, we need to further reduce them to $L M C$-expressions of subgraphs of $B$.

Theorem 5.3 [105]. Let $B_{1}, B_{2}, \cdots, B_{t}$ be $t$ mutually disjoint $B H \mathrm{~s}$, or $B H$-fragments, and $B=B_{1} \cup B_{2} \cup \cdots \cup B_{t}$. Then

$$
R(B)=R\left(B_{1} \cup B_{2} \cup \cdots \cup B_{t}\right)=\sum_{i=1}^{t} \frac{K(B)}{K\left(B_{i}\right)} R\left(B_{i}\right) .
$$

Theorem 5.4 [105]. Let $B_{1}, B_{2}, \cdots, B_{t}$ be the normal components of an essentially disconnected $B H$ or a $B H$ fragment B. Then

$$
R(B)=R\left(B^{*}\right)=\sum_{i=1}^{t} \frac{K(B)}{K\left(B_{i}\right)} R\left(B_{i}\right) .
$$

Theorem 5.5 [105]. Let $B$ be a $B H$ which contains no crown (see Fig. 10 (1)) as its subgraph. Then for any edge $e=u v$ of $B$, each of $L M$-conjugated circuits of $B_{e}^{*}\left(B_{\bar{e}}^{*}\right)$ is also a minimal in $B_{e}\left(B_{\bar{e}}\right)$.

Theorem 5.6 [105]. Let $B$ be a normal $B H$, which contains no crown as its subgraph. Then, for any edge $e$ of $B$,

$$
\begin{aligned}
& R\left(B_{e}\right)=R^{\prime}\left(B_{e}\right)+R\left(B_{\bar{e}}^{*}\right), R\left(B_{\bar{e}}\right)=R^{\prime}\left(B_{e}\right)+R\left(B_{\bar{e}}^{*}\right), \\
& R(B)=R\left(B_{e}^{*}\right)+R\left(B_{\bar{e}}^{*}\right)+R^{\prime}\left(B_{e}\right)+R^{\prime}\left(B_{\bar{e}}\right) .
\end{aligned}
$$

Theorem 5.7 [105]. Let $e$ be an edge on the boundary of a benzenoid hydrocarbon $B$, and let $S_{h}\left(B_{e^{\prime}}\right)\left(S_{h}\left(B_{\bar{e}}{ }^{\prime}\right)\right)$ be

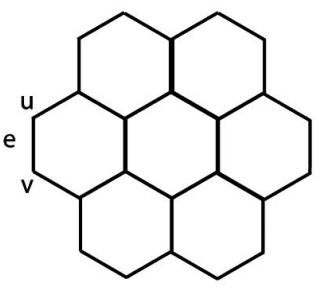

A Crown B

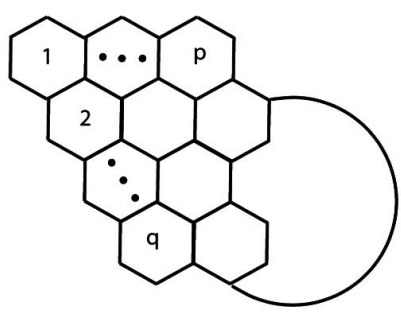

$p \geqslant 1, \quad q \geqslant 1, \quad p+q \geqslant 3$

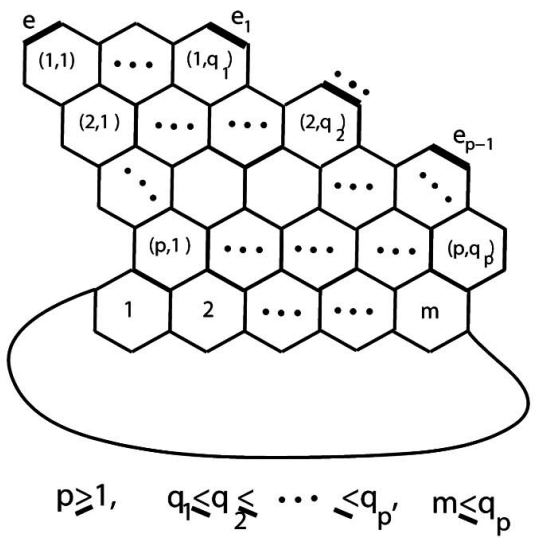

(3)

Fig. (10). (1) A crown $B$. (2) A recursive edge $e$ in a $B H \quad B$ with a local struction. (3) Recursive edges $e, e_{1}, e_{2}, \cdots, e_{p-1}$ in a $B H \quad B$ with a local struction. 
the set of rings in $B_{e^{\prime}}\left(B_{\bar{e}^{\prime}}{ }^{\prime}\right.$. Let $C_{s}\left(B_{e}\right)\left(C_{s}\left(B_{\bar{e}}\right)\right)$ denote the set of minimal conjugated circuits of a ring $s$ in $B_{e}$ $\left(B_{\bar{e}}\right)$. Then for $s \in S_{h}\left(B_{e^{\prime}}\right), R_{s}\left(B_{e}\right)=\sum_{C \in C_{s}\left(B_{e}\right)} K(B-C) R_{(|C|-2) / 4}$, for $s \in S_{h}\left(B_{\bar{e}}{ }^{\prime}, R_{s}\left(B_{\bar{e}}\right)=\sum_{C \in C_{s}\left(B_{\bar{e}}\right)} K(B-C) R_{(|C|-2) / 4}\right.$, where $|C|$ denotes the length of $C, K(B-C)$ is the number of Kekulé structures of $B-C$.

Definition 5.4 [105]. Let $e$ be an edge on the boundary of a benzenoid hydrocarbon $B$. If $B$ and $e$ satisfy one of the following conditions: (1) $B$ contains no crown (see Fig. 10 (1)) as its subgraph; (2) $B$ contains a local structure as shown in Fig. 10 (2), and $e$ is the marked edge; (3) $B$ contains a local structure as shown in Fig. 10 (3), and $e$ is the marked edge; then $e$ is said to be a recursive edge of $B$.

Theorem 5.8 [105]. Let $B$ be a $B H$ which contains a recursive edge $e$ on the boundary of $B$. Then

$$
\begin{aligned}
& R(B)=R^{*}\left(B_{e}\right)+R^{*}\left(B_{\bar{e}}\right)+R^{\prime}\left(B_{e}\right)+R^{\prime}\left(B_{\bar{e}}\right) \\
& 1.8 \mathrm{~cm}=R\left(B_{e}^{*}\right)+R\left(B_{\bar{e}}^{*}\right)+\sum_{s \in S_{h}\left(B_{e^{\prime}}\right)} \sum_{C \in C_{s}\left(B_{e}\right)} K(B-C) R_{(|C|-2) / 4} \\
& 2.3 \mathrm{~cm}+\sum_{s \in S_{h}\left(B_{\bar{e}}\right)} \sum_{C \in C_{s}\left(B_{\bar{e}}\right)} K(B-C) R_{(|C|-2) / 4}
\end{aligned}
$$

Definition 5.5 [105]. Let $C$ be a minimal conjugated circuit of a ring $s$ of a benzenoid hydrocarbon $B$, and let $s^{\prime}$ be a hexagon of $B$ for which $C \cap s^{\prime} \neq \varnothing$ and the interior of $s^{\prime}$ is contained in the exterior of $C$. If $C^{\prime}=C \cap s^{\prime}$ (the symmetry difference of edge sets of $C$ and $s^{\prime}$ ) is also a minimal conjugated circuit of $s$, then we say $C^{\prime}$ is obtained from $C$ by a extension and $s^{\prime}$ is a extendible hexagon of $C$. For a ring $s$ in $B_{e}\left(B_{\bar{e}}\right)$, a minimal conjugated circuit $C$ of $s$ in $B_{e}\left(B_{\bar{e}}\right)$ is said to be minimum if $C$ has the smallest length and $B[C]$ contains a smallest number of hexagons.

Theorem 5.9 [105]. Let $e$ be a recursive edge of a benzenoid hydrocarbon $B$, and let $s$ be a ring in $B_{e^{\prime}}\left(B_{\bar{e}}{ }^{\prime}\right)$. Let $C$ be a minimal conjugated circuit of $s$ in $B_{e}\left(B_{\bar{e}}\right)$ which is not minimum. Then $C$ can be obtained from another minimal conjugated circuit of $s$ in $B_{e}\left(B_{\bar{e}}\right)$ by an extension.

Procedure 5.1 [105]. Let $e$ be a recursive edge of a benzenoid hydrocarbon $B$, and $s$ a ring in $B_{e}\left(B_{\bar{e}}\right)$. Let $C^{*}$ be a unique minimum conjugated circuit of $s$ in $B_{e}$ $\left(B_{\bar{e}}\right)$.

(1) Set $S_{0}=\left\{C^{*}\right\}, S_{i}=S_{0}$.

(2) For every minimal conjugated circuit $C_{i}$ in $S_{i}$, find all extendible hexagons of $C_{i}$, extend $C_{i}$ to new minimal conjugated circuits, and set them to $S_{i+1}$. If $S_{i+1}=\varnothing$, then go to (4). Otherwise set $i+1 \rightarrow i$, go to (2).

$$
\text { Set } C_{s}\left(B_{e}\right)=\bigcup_{j=1}^{i} S_{j} \quad\left(C_{s}\left(B_{\bar{e}}\right)=\bigcup_{j=1}^{i} S_{j}\right) .
$$

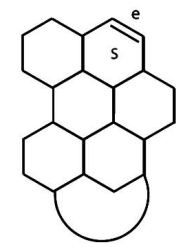

$B_{e}$

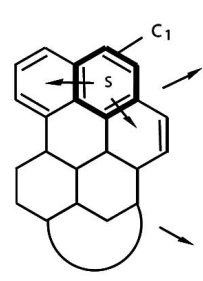

7

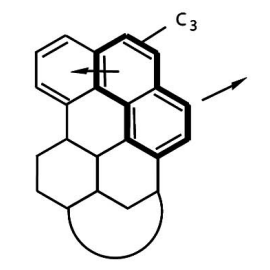

$C_{S}(B)=\left\{C_{1}, C_{2}, \cdots, C_{5}\right\}$
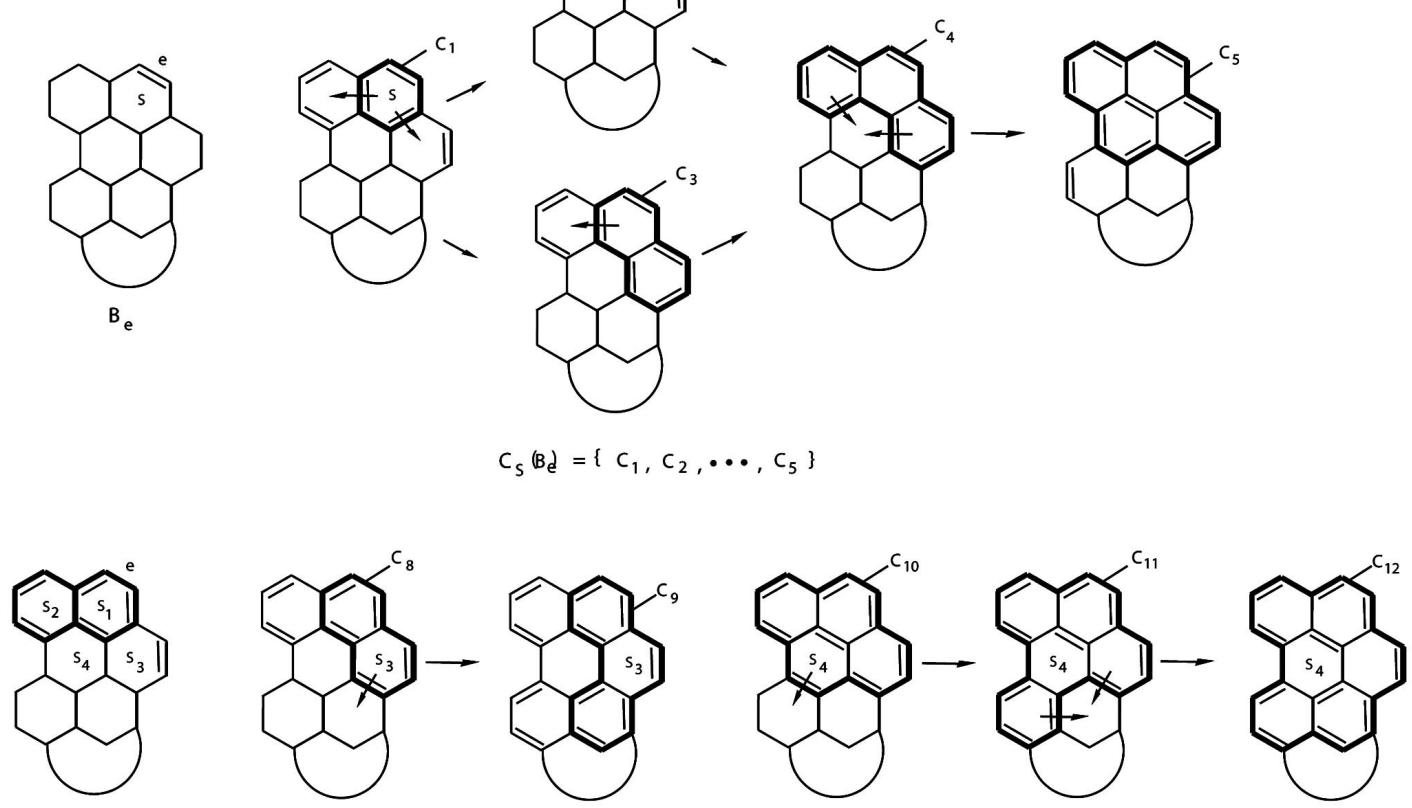

$\mathrm{B}_{\overline{\mathrm{e}}}$

$$
C_{6}=s_{1}, C_{7}=s_{1} \Delta s_{2}, C_{s_{1}} B \frac{1}{e}=\left\{C_{6}\right\}, C_{s_{2}}\left(B \frac{1}{e}=\left\{C_{7}\right\}, C_{s_{3}}=\left\{C_{8}, C_{9}\right\}, C_{S_{4}}\left(B \frac{1}{e}=\left\{C_{10}, C_{11}, C_{12}\right\}\right.\right.
$$

Fig. (11). An example for application of procedure 1 . 
Fig. 11.

An example of application of Procedure 5.1 is shown in

5.3. $L M$-Conjugated Circuit Polynomials in Catacondensed Benzenoid Hydrocarbons

In a general case, a catacondensed benzenoid hydrocarbon (cata- $B H$ ) $B$ has the construction shown in Fig. (12), where $B_{1}, B_{2}, B_{3}, \cdots, B_{15}$ are subgraphs of $B$, each of which is a cata- $B H$. Particularly, if $n_{2}=n_{3}=0, B$ becomes a straight cata- $B H$.

Theorem 5.10 [107]. Let $B=B_{a}(n)$ denote the straight cata- $B H$ with $n$ hexagons (see Fig. (13a)). Then $R(B)=2 \sum_{i=1}^{n}(n+1-i) R_{i}$.

Theorem 5.11 [107]. Let $B$ be a cata- $B H$ shown in Fig. (12). Then

$$
\begin{aligned}
& R(B)=R\left(B_{1}\right)+\left(n_{1}-1\right)\left[K\left(B_{3}\right) R\left(B_{2}\right)+\right. \\
& \left.K\left(B_{2}\right) R\left(B_{3}\right)\right]+2 K\left(B_{2}\right) K\left(B_{3}\right) \sum_{i=1}^{n-1}(n-i) R_{i} \\
& +\left[\prod_{j=4}^{7} K\left(B_{j}\right)\right]\left[\sum_{i=2}^{n_{1}} \sum_{j=0}^{n_{2}-1} \sum_{k=0}^{n_{3}-1} R_{i+j+k}+\right. \\
& \sum_{i=2}^{n_{1}} R_{i}+\left[\prod_{j=6}^{11} K\left(B_{j}\right)\right] \sum_{i=2}^{n_{1}} \sum_{k=0}^{n_{3}-1} R_{i+n_{2}+k} \\
& +\left[\prod_{j=4,5,12}^{15} K\left(B_{j}\right)\right] \sum_{i=2}^{n_{1}} \sum_{j=0}^{n_{2}-1} R_{i+j+n_{3}}+\left[\prod_{j=8}^{15} K\left(B_{j}\right)\right] \sum_{i=2}^{n_{1}} R_{i+n_{2}+n_{3}}
\end{aligned}
$$

where, if $n_{2}=0\left(n_{3}=0\right)$, then $K\left(B_{j}\right)=1$ for $j=2,4,5(j=3,6,7), \quad K\left(B_{j}\right)=0 \quad$ for $\quad j=8,9,10,11$ $(j=12,13,14,15)$, and

$\sum_{i=2}^{n_{1}} \sum_{j=0}^{n_{2}-1} \sum_{k=0}^{n_{3}-1} R_{i+j+k}=\sum_{i=2}^{n_{1}} \sum_{k=0}^{n_{3}-1} R_{i+k}\left(\sum_{i=2}^{n_{1}} \sum_{j=0}^{n_{2}-1} R_{i+j}\right)$.

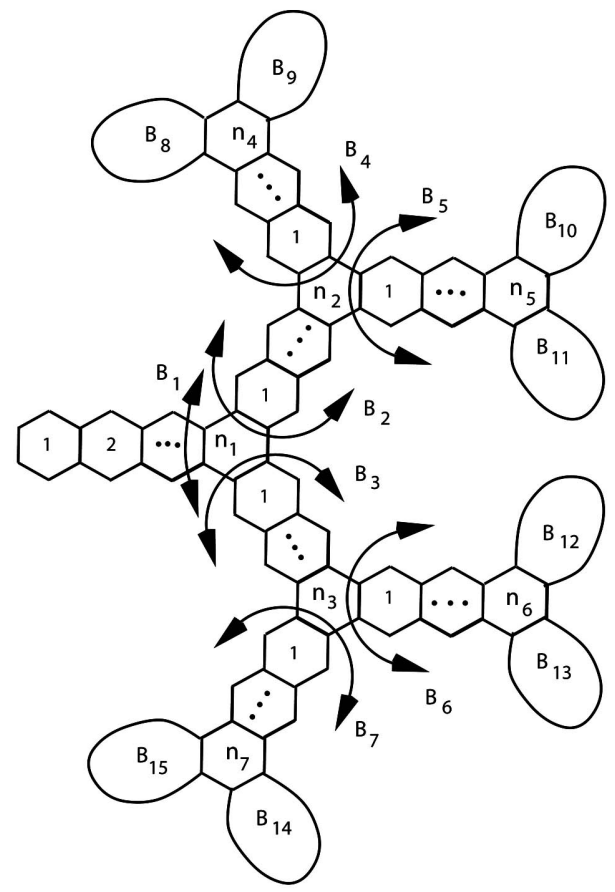

Fig. (12). The construction of a catacondensed benzenoid hydrocarbon.
Corollary 5.1 [107]. Let $B=B_{u}\left(m_{1}, m_{2}, \cdots, m_{t}\right)$ denote an unbranched cata- $B H$ as shown in Fig. (14). Then

$R(B)=m_{1} R\left(B_{2}\right)+R\left(B_{3}\right)+\sum_{i=1}^{m_{1}-1}\left[2 K\left(B_{2}\right)\left(m_{1}-i\right)+K\left(B_{3}\right)\right] R_{i}+2 K\left(B_{3}\right)$

$\sum_{i=1}^{m_{2}} R_{i}+K\left(B_{3}\right)\left[\sum_{i=2}^{m_{1}} \sum_{j=0}^{m_{2}-1} R_{i+j}+R_{m_{1}}-R_{1}\right]+K\left(B_{4}\right)$

$\left[\sum_{i=m_{2}+2}^{m_{1}+m_{2}} R_{i}+2 R_{m_{2}+1}+\sum_{i=m_{2}+2}^{m_{2}+m_{3}} R_{i}\right]+K\left(B_{5}\right) R_{m_{2}+m_{3}+1}$

where, if $m_{2}=0\left(m_{3}=0\right)$, then $K\left(B_{j}\right)=1$ for $j=2,3(j=3,4)$ and $K\left(B_{j}\right)=0$ for $j \geq 4(j \geq 5)$.

By Theorem 5.10 and Corollary 5.1, we can easily obtain the following calculation formulae for enumerating the $L M$-conjugated circuits of the cata- $B H \mathrm{~s}$ in Fig. (13).

Corollary 5.2 [107]. Let $B_{b}(n), n \geq 2$, be the cata- $B H$ shown in Fig. (13b). Then

$R\left(B_{b}(n)\right)=(6 n-2) R_{1}+4(n-1) R_{2}+\sum_{i=3}^{n}(4 n+3-4 i) R_{i}+R_{n+1}$.

Corollary 5.3 [107]. Let $B_{c}(n), n \geq 1$, be the cata- $B H$ shown in Fig. (13c). Then

$R_{c}\left(B_{c}(n)\right)=4(4 n+1) R_{1}+8 n R_{2}+4 \sum_{i=3}^{n}(2 n+3-2 i) R_{i}+6 R_{n+1}+2 R_{n+2}$.

Corollary 5.4 [107]. Let $B_{d}(n), n \geq 2$, be the cata- $B H$ shown in Fig. (13d). Then

$R\left(B_{d}(n)\right)=2(8 n-3) R_{1}+2(4 n-5) R_{2}+$

$\sum_{i=3}^{n}(8 n+5-8 i) R_{i}+3 R_{n+1}+R_{n+2}$

Corollary 5.5 [107]. Let $B_{e}(n), n \geq 1$, be the cata- $B H$ shown in Fig. (13e). Then

$$
\begin{aligned}
& R\left(B_{e}(n)\right)=96 n R_{1}+8 R_{2}+8 \sum_{i=2}^{n} \\
& (4 n+5-4 i) R_{i}+26 R_{n+1}+12 R_{n+2}+2 R_{n+3} .
\end{aligned}
$$

Corollary 5.6 [107]. Let $B_{f}(n), n \geq 3$, be the cata- $B H$ shown in Fig. (13f). Then

$R\left(B_{f}(n)\right)=\sum_{i=0}^{n-1} F_{i}\left(2 F_{n-i-1} R_{1}+2 F_{n-i-2} R_{2}+F_{n-i-3} R_{3}\right)$,

where $F_{i}=F_{i-1}+F_{i-2}$ is Fibonacci; ${ }^{-}$s number, $F_{0}=F_{1}=1$ and $F_{i}=0$ for $i \leq-1$.

Concerning more general cases, we give the following examples.

Corollary 5.7 [107]. Let $B_{u}\left(m_{1}, m_{2}\right)$ be an unbranched cata- $B H$ (see Fig. 14). Then

$$
\begin{aligned}
& R\left(B_{u}\left(m_{1}, m_{2}\right)\right)=\sum_{i=1}^{m_{1}}\left[2\left(m_{2}+1\right)\left(m_{1}-i\right)+1\right] R_{i}+2 \\
& \sum_{i=1}^{m_{2}}\left[m_{1}\left(m_{2}+1-i\right)+1\right] R_{i} \quad 4 c m+\sum_{i=m_{2}+2}^{m_{1}+m_{2}} R_{i}+ \\
& \sum_{i=2}^{m_{1}} \sum_{j=0}^{m_{2}-1} R_{i+j}-R_{1}+2 R_{m_{2}+1}
\end{aligned}
$$

Corollary 5.8 [107]. Let $B_{u}\left(m_{1}, m_{2}, m_{3}\right)$ be an unbranched cata- $B H$ (see Fig. 14). Then 


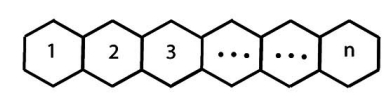

(a) B (n)

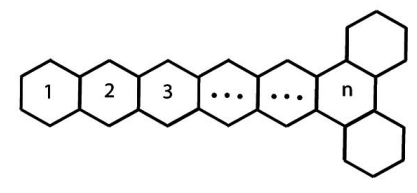

(d) $B$ (n)

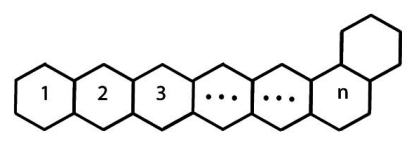

(b) $B_{\text {(b) }}$

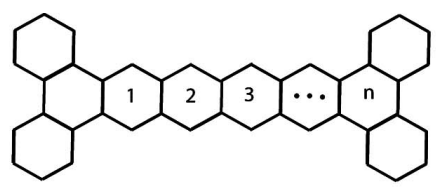

(e) $B$ (n)

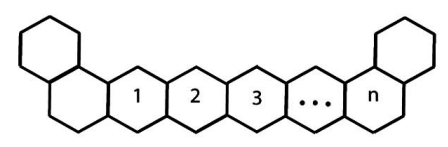

(c) $B$ (n)

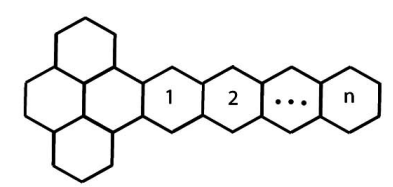

(g) $B$ ( $n$ )

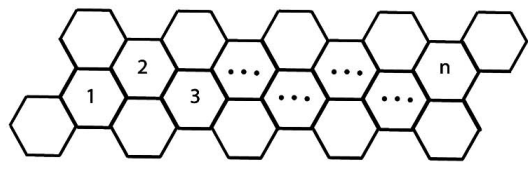

(i) $B$ (n)

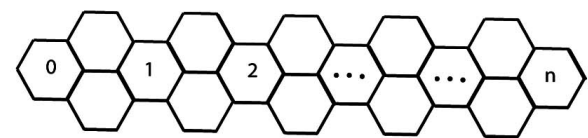

(h) $B_{h}(n)$

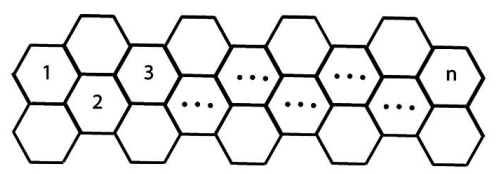

(j) $B$ (n)

Fig. (13). Some families of benzenoid hydrocarbons.

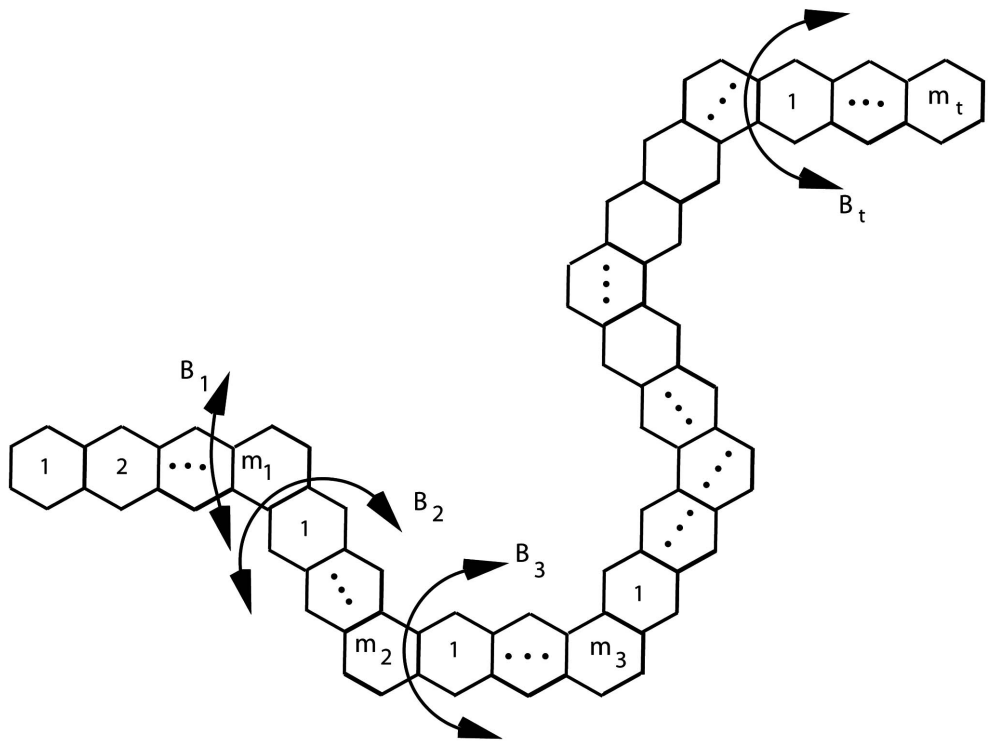

Fig. (14). An unbranched catacondensed benzenoid hydrocarbon B.

$$
\begin{array}{ll}
R\left(B_{u}\left(m_{1}, m_{2}, m_{3}\right)\right) & +\left(m_{3}+1\right) \sum_{i=2}^{m_{1}} \sum_{j=0}^{m_{2}-1} R_{i+j}+m_{1} \sum_{i=2}^{m_{2}} \sum_{j=0}^{m_{3}-1} R_{i+j}+ \\
=\sum_{i=1}^{m_{1}-1}\left\{2\left[m_{2}\left(m_{3}+1\right)+1\right]\left(m_{1}-i\right)+\left(m_{3}+1\right)\right\} R_{i} & \sum_{i=m_{2}+2}^{m_{1}+m_{2}} R_{i}+\sum_{i=m_{2}+2}^{m_{2}+m_{3}} R_{i} \\
+\sum_{i=1}^{m_{2}}\left[2 m_{1}\left(m_{3}+1\right)\left(m_{2}-i\right)+m_{1}+2 m_{3}+2\right] R_{i}+2 & +m_{1} \sum_{i=m_{3}+2}^{m_{2}+m_{3}} R_{i}-\left(m_{1}+m_{3}+1\right) R_{1}+\left(m_{3}+1\right) \\
\sum_{i=1}^{m_{3}^{3}}\left[\left(m_{3}+1-i\right)\left(m_{1} m_{2}+1\right)+m_{1}\right] R_{i} & R_{m_{1}}+2 R_{m_{2}+1}+2 m_{1} R_{m_{3}+1}+R_{m_{2}+m_{3}+1}
\end{array} . .
$$




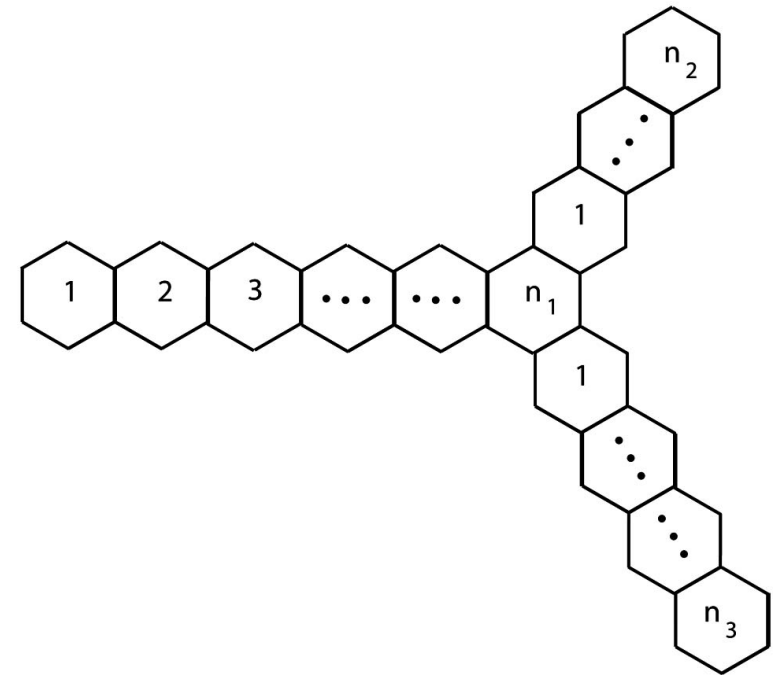

Fig. (15). A catacondensed benzenoid hydrocarbon $B_{Y}\left(n_{1}, n_{2}, n_{3}\right)$ consisting of three straight catacondensed benzenoid hydrocarbons.

Corollary 5.9 [107]. Let $B_{Y}\left(n_{1}, n_{2}, n_{3}\right)$ denote the cata$B H$ shown in Fig. (15). Then

$R\left(B_{Y}\left(n_{1}, n_{2}, n_{3}\right)\right)$

$=\sum_{i=1}^{n_{1}}\left[2\left(n_{2}+1\right)\left(n_{3}+1\right)\left(n_{1}-i\right)+1\right] R_{i} \ddot{+} \sum_{i=1}^{n_{2}}$

$\left[2 n_{1}\left(n_{3}+1\right)\left(n_{2}+1-i\right)+1\right] R_{i}$

$+\sum_{i=1}^{n_{3}} 2\left[n_{1}\left(n_{2}+1\right)\left(n_{3}+1-i\right)+1\right] R_{i}+\sum_{i=2}^{n_{1}} \sum_{j=0}^{n_{2}-1} \sum_{k=0}^{n_{3}-1} R_{i+j+k}$

$+\sum_{i=2}^{n_{1}} \sum_{j=0}^{n_{2}-1} R_{i+j+n_{3}}+\sum_{i=2}^{n_{1}} \sum_{k=0}^{n_{3}-1} R_{i+n_{2}+j}+\sum_{i=2}^{n_{2}-1} \sum_{j=0}^{n_{3}-1} R_{i+j}$

$+\sum_{i=n_{3}+1}^{n_{1}+n_{2}+n_{3}} R_{i}-2 R_{1}+R_{n_{2}+1}+R_{n_{3}+1}$.

\section{4 $L M$-Conjugated Circuit Polynomials in Some Families of Structurally Related Pericondensed Benzenoid Hydrocarbons}

To enumerate $L M$-conjugated circuits in some families of structurally related pericondensed benzenoid hydrocarbons, we need to use Theorem 5.8 and procedure 5.1 , and often need to deal with several recursive relations of several families of structurally related subgraphs for a family of structurally related peri- $B H \mathrm{~s}$. We will give some results but omit the operation processes.

We first give the recursive formulae for enumeration of $L M$-conjugated circuits of the peri-BHs in Fig. (13).

Corollary 5.10 [107]. Let $B_{g}(n)$ be the $B H$ shown in Fig. (13g). Then

$$
\begin{aligned}
& R\left(B_{g}(n)\right)=2 \sum_{i=1}^{n}(5 n+8-5 i) R_{i}+(10 n+6) R_{1}+4(n+1) \\
& R_{2}+(n+2) R_{3} \\
& +6 R_{n+1}+4 R_{n+2}+2 R_{n+3} .
\end{aligned}
$$

Corollary 5.11 [107]. Let $B_{h}(n)$ be the $B H$ shown in Fig. (13h). Then

$$
\begin{aligned}
& R\left(B_{h}(n)\right)=2 \sum_{i=0}^{n-1} R\left(B_{h}(i)\right)+2\left[2 \sum_{i=-1}^{n-2}(n-1-i)\right. \\
& \left.K\left(B_{h}(i)\right)+K\left(B_{h}(n-1)\right)\right] R_{1} \\
& +8 \sum_{i=-1}^{n-2} K\left(B_{h}(i)\right) R_{2}+2\left[3 \sum_{i=-1}^{n-3}(n-2-i)\right. \\
& \left.K\left(B_{h}(i)\right)+2 K\left(B_{h}(n-2)\right)\right] R_{3} \\
& +8 \sum_{i=-1}^{n-3} K\left(B_{h}(i)\right) R_{4}+4 \sum_{i=-1}^{n-4}(n-3-i) K\left(B_{h}(i)\right) R_{5},
\end{aligned}
$$

where

$$
\begin{aligned}
& R\left(B_{h}(0)\right)=2 R_{1}, K\left(B_{h}(n)\right)=2 \sum_{i=-1}^{n-1} K\left(B_{h}(i)\right), K\left(B_{h}(0)\right)=2, \\
& \left.K\left(B_{h}(-1)\right)=1, \text { and } K\left(B_{h}(i)\right)=0 \text { for } i\right] \leq-2 .
\end{aligned}
$$

Corollary 5.12 [107]. Let $B_{i}(n)$ be the $B H$ shown in Fig. (13i). Then, for $n=2 p$,

$$
\begin{aligned}
& R\left(B_{i}(n)\right)=2 R\left(B_{i}(n-1)\right)+\sum_{j=-1}^{(n / 2)-2} R\left(B_{i}(2 j+1)\right) \\
& +2\left[\sum_{j=-1}^{(n / 2)-2}(n / 2-1-j) K\left(B_{i}(2 j+1)\right)+K\left(B_{i}(n-1)\right)\right] R_{1}+2 \\
& \sum_{j=-1}^{n-2} K\left(B_{i}(j)\right) R_{2} \\
& +\left\{\sum_{j=-1}^{(n / 2)-2}\left(\frac{n}{2}-1-j\right)\left[2 K\left(B_{i}(2 j)\right)+K\left(B_{i}(2 j+1)\right)\right]+\right. \\
& \left.K\left(B_{i}(n-2)\right)\right\} R_{3} \\
& +\left\{\sum_{j=-1}^{(n / 2)-2}\left[2 K\left(B_{i}(2 j)\right)+\left(\frac{n}{2}-2-j\right) K\left(B_{i}(2 j+1)\right)\right]+\right. \\
& \left.K\left(B_{i}(n-3)\right)\right\} R_{4}
\end{aligned}
$$

for $n=2 p+1$,

$$
\begin{aligned}
& R\left(B_{i}(n)\right)=2 R\left(B_{i}(n-1)\right)+\sum_{j=0}^{(n-3) / 2} R\left(B_{i}(2 j)\right) \\
& +2\left[\sum_{j=-1}^{(n-3) / 2}\left(\frac{n-1}{2}-j\right) K\left(B_{i}(2 j)\right)+K\left(B_{i}(n-1)\right)\right] R_{1}+2 \\
& \sum_{j=-2}^{n-2} K\left(B_{i}(j)\right) R_{2} \\
& +\left\{\sum _ { j = - 1 } ^ { ( n - 3 ) / 2 } \left[\left(\frac{n-1}{2}-j\right) K\left(B_{i}(2 j)\right)+2\left(\frac{n-1}{2}-1-j\right)\right.\right. \\
& \left.\left.K\left(B_{i}(2 j+1)\right)\right]+K\left(B_{i}(n-2)\right)\right\} R_{3} \\
& +\left\{\sum_{j=-1}^{(n-5) / 2}\left[\frac{n-1}{2}-1-j\right) K\left(B_{i}(2 j)\right)+2 K\left(B_{i}(2 j+1)\right)\right]+ \\
& \left.K\left(B_{i}(n-3)\right)\right\} R_{4}
\end{aligned}
$$

where

$R\left(B_{i}(0)\right)=8 R_{1}, R\left(B_{i}(-1)\right)=2 R_{1}$,

$K\left(B_{i}(n)\right)=K\left(B_{i}(n-1)\right)+\sum_{j=-1}^{(n / 2)-1} K\left(B_{i}(2 j+1)\right)$ for $n=2 p$,

$K\left(B_{i}(n)\right)=K\left(B_{i}(n-1)\right)+\sum_{j=-1}^{(n-1) / 2} K\left(B_{i}(2 j)\right)$ for $n=2 p+1$,

$K\left(B_{i}(0)\right)=4, K\left(B_{i}(-1)\right)=2, K\left(B_{i}(-2)\right)=1$ and $K\left(B_{i}(j)\right)=0$

for $j \leq-3$. 
Corollary 5.13 [107]. Let $B_{j}(n)$ be the $B H$ shown in Fig. (13j). Then

$$
\begin{aligned}
& R\left(B_{j}(n)\right)=R\left(B_{j}(n-1)\right)+2 R\left(B_{j}(n-2)\right)+\sum_{i=1}^{n-3} R\left(B_{j}(i)\right) \\
& +2\left[\sum_{i=-1}^{n-3}(n-1-i) K\left(B_{j}(i)\right)+2 K\left(B_{j}(n-2)\right)\right] R_{1} \\
& +2\left[2 \sum_{i=-1}^{n-3} K\left(B_{j}(i)\right)+K\left(B_{j}(n-2)\right)\right] R_{2} \\
& +\left[\sum_{i=-1}^{n-5}(n-1-i) K\left(B_{j}(i)\right)+4 K\left(B_{j}(n-3)\right)+4 K\left(B_{j}(n-4)\right)\right] R_{3} \\
& +\left[\sum_{i=-1}^{n 5}(n-1-i) K\left(B_{j}(i)\right)+4 K\left(B_{j}(n-4)\right)\right] R_{4},
\end{aligned}
$$

where

$$
\begin{aligned}
& R\left(B_{j}(n)\right)=0 \text { for } n \leq 0, \\
& K\left(B_{j}(n)\right)=K\left(B_{j}(n-2)\right)+\sum_{i=-1}^{n-1} K\left(B_{j}(i)\right), \\
& K\left(B_{j}(n)\right)=1 \text { for } n=0,-1, \text { and } K\left(B_{j}(n)\right)=0 \text { for } n \leq-2 .
\end{aligned}
$$

Finally, for the two families of peri- $B H$ s shown in Fig. (16), we give the recursive formulae for enumeration of their $L M$-conjugated circuit polynomials.

Corollary 5.14 [107]. Let $B_{k}(n)$ be the $B H$ shown in Fig. (16(1)). Then

$$
\begin{aligned}
& R\left(B_{k}(n)\right)=5 R\left(B_{k}(n-1)\right)+4 \sum_{i=1}^{n-2} R\left(B_{k}(i)\right) \\
& +2\left[4 \sum_{i=0}^{n-2}(n-i) K\left(B_{k}(i)\right)+5 K\left(B_{k}(n-1)\right)+n\right] R_{1} \\
& +2\left[\sum_{i=0}^{n-2}(8 n-7-8 i) K\left(B_{k}(i)\right)+2 K\left(B_{k}(n-1)\right)+2 n\right] R_{2} \\
& +\left[4 \sum_{i=0}^{n-2}(n+2-i) K\left(B_{k}(i)\right)+K\left(B_{k}(n-1)\right)+n+2\right] R_{3} \\
& +\left[2 \sum_{i=0}^{n-3}(2 n-3-2 i) K\left(B_{k}(i)\right)+3 K\left(B_{k}(n-2)\right)+n-1\right] R_{4},
\end{aligned}
$$

where

$$
\begin{aligned}
& R\left(B_{k}(0)\right)=0, \quad K\left(B_{k}(n)\right)=5 K\left(B_{k}(n-1)\right)+4 \sum_{i=0}^{n-2} K\left(B_{k}(i)\right)+1, \\
& K\left(B_{k}(0)\right)=1 \text { and } K\left(B_{k}(i)\right)=0 \text { for } i \leq-1 .
\end{aligned}
$$

Corollary 5.15 [107]. Let $B_{l}[m, n]$ be the $B H$ shown in Fig. (16(2)). Then

$$
R\left(B_{l}[m, n]\right)=R\left(B_{l}[m-1, n]\right)+R\left(B_{l}[m, n-1]\right)
$$

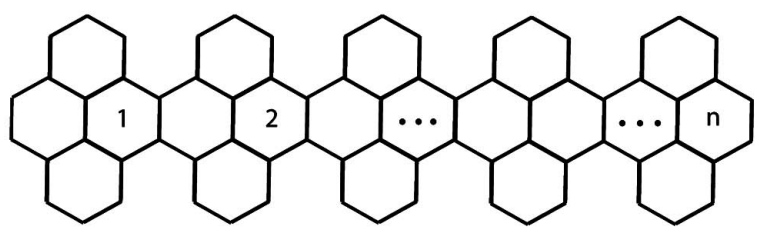

(1)

Fig. (16). Two families of pericondensed benzenoid hydrocarbons.

$$
+2 \sum_{i=1}^{m} \sum_{j=1}^{n} K\left(B_{l}[m-i, n-j]\right) K\left(B_{l}[i-1, j-1]\right) R_{i+j-1},
$$

where

$$
\begin{aligned}
& K\left(B_{l}[m, n]\right)=K\left(B_{l}[m-1, n]\right)+\left(B_{l}[m, n-1]\right), \\
& K\left(B_{l}[m, 0]\right)=K\left(B_{l}[0, n]\right)
\end{aligned}
$$

\subsection{Analytical Expressions for the Count of $L M$ -} Conjugated Circuits of Benzenoid Hydrocarbons

Although the LMCC -polynomials of several classes of benzenoid hydrocarbons can be directly obtained from the LMCC -polynomials and the Kekule structure counts of some subgraphs by the above recursive method [105, 108], there are still some classes of benzenoid hydrocarbons whose $L M C$ - expressions cannot be obtained by the recursive method, e.g., the benzenoid hydrocarbons shown in Fig. (17). So we need to investigate a new method to calculate such $L M C$ - expressions.

In ref. [108], Guo, Randic', and Klein investigated further properties of $L M$-conjugated circuits and different contributions of $L M$-conjugated circuits having different shapes to $R(B)$ and gave a new method for calculating the $L M C C$-polynomials of polycyclic benzenoid hydrocarbons, which is based on the counts of Kekule structures of selected subgraphs of benzenoid hydrocarbons. By using the method, the $L M C C$-polynomials of any benzenoid hydrocarbon can be obtained.

Theorem 5.2 establishes the theoretical basis of the partition of the $L M C C$-polynomial of $\mathrm{B}$ into the $L M C C$ polynomials of rings of $B$. The $L M C C$-polynomial of a ring $s$ of a benzenoid hydrocarbon $B$, denoted by $R_{s}(B)$, is the summation of all the minimal conjugated circuits of $s$, one for each Kekulé structure of $B$. Note that $R_{s}(B)$ is just the contribution of $s$ to $R(B)$, and $R(B)=\sum_{s}^{B} R_{s}(B)$ $\left(R_{s}(B)=0\right.$ for $\left.s \notin B^{*}\right)$. And, $R_{s}(B)$ may also be denoted by a sequence of numbers (ring code) $\left(r_{1}(s), r_{2}(s), \cdots, r_{n}(s), \cdots\right)$, where $r_{n}(s)$ is the coefficient of the term $R_{n}$ in $R_{s}(B)$.

Hence, the count of $L M$-conjugated circuits of a benzenoid hydrocarbon $B$ is reduced to calculations of $R_{s}(B)$ for every ring $s$ of $B$. To calculate $R_{s}(B)$, we need

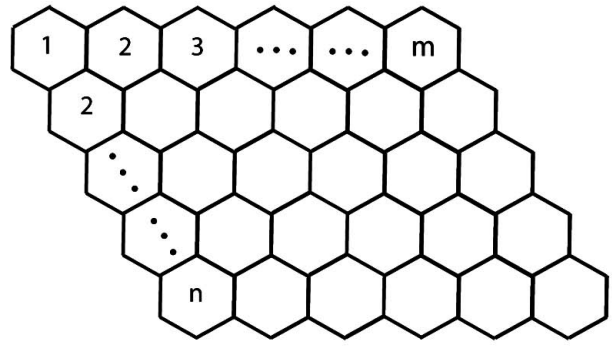

(2) 


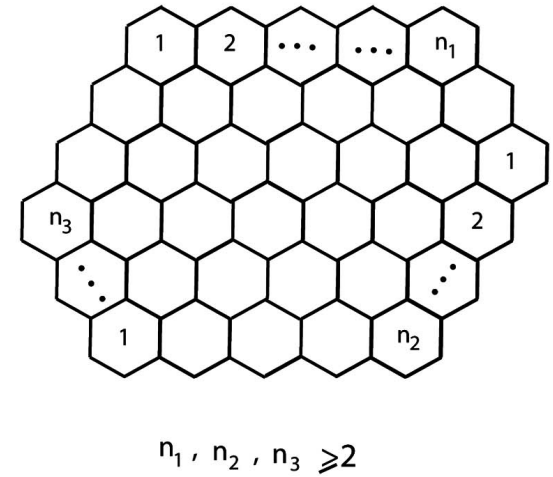

Fig. (17). Two families of pericondensed benzenoid hydrocarbons.

to investigate further properties of minimal conjugated circuits of benzenoid hydrocarbons.

For a Kekulé structure $K_{i}$, of a benzenoid hydrocarbon $B$, Theorem 5.1 gives all possible configurations of a minimal $K_{i}$-conjugated circuit $C$ of a ring $s$ of $B$ (see Fig. 9). We call a configuration of $C$ a minimal configuration ( $M$-configuration) with respect to (w.r.t.) the ring $s$. Conversely, for a circuit in $B$ which has an $M$ configuration w.r.t. a ring $s$, we have the following:

Lemma 5.1 [108]. Let $C$ be a conjugated circuit with an $M$-configuration w.r.t. a ring $s$ in a benzenoid hydrocarbon $B$. Then, for any Kekulé structure $K_{i^{\prime}}$ in $B-C$, there is exactly one Kekulé structure $K_{i}$ in $B$ for which $K_{i^{\prime}} \subset K_{i}$, and $C$ is a minimal $K_{i}$-conjugated circuit of $s$ in $B$.

By Lemma 5.1, we can give a new definition of a minimal conjugated circuit of a ring of $B$, which is independent of a Kekulé structure $K_{i}$ of $B$.

Definition 5.6 [108]. A conjugated circuit $C$ of a benzenoid hydrocarbon $B$ is said to be minimal if $C$ has an $M$-configuration w.r.t. a ring $s$ in $B . C$ is also said to be a minimal conjugated circuit of $s$ in $B$.

Corollary 5.16 [108]. Let $C$ be a circuit with an $M$ configuration w.r.t. a ring $s$ in a benzenoid hydrocarbon $B$. Then $C$ corresponds to exactly $K(B-C)(=K(B-B[C]))$ Kekulé structures for each of which $C$ is a minimal conjugated circuit of $s$ in $B$.

Note that Corollary 5.16 cannot be used to calculate $R_{s}(B)$. In general cases, the coefficient $r_{n}(s)$ of the term $R_{n}$ in $R_{s}(B)$ is not equal to $\sum_{C_{4 n+2}} K\left(B-C_{4 n+2}\right)$, here $C_{4 n+2}$ is a minimal conjugated circuit of $s$ with size $4 n+2$, because for a Kekulé structure $K_{i}$ and a ring $s$ of $B$, a

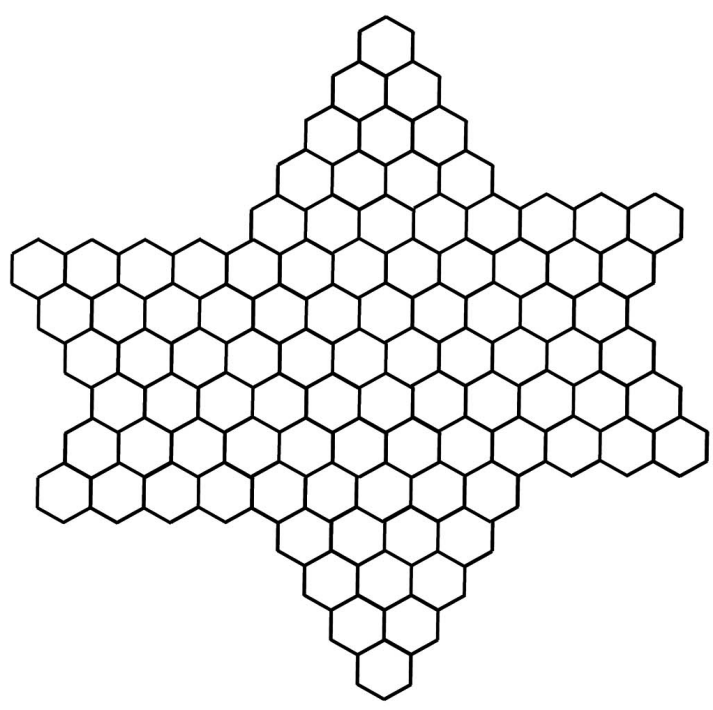

minimal $K_{i}$-conjugated circuit $C$ of $s$ may be not unique (see Fig. 18), but calculating $R_{s}(B)$ requires one to take exactly one minimal conjugated circuit of $s$ for each Kekulé structure. Therefore, we need to investigate the properties of non-unique minimal conjugated circuits of a ring in $B$.

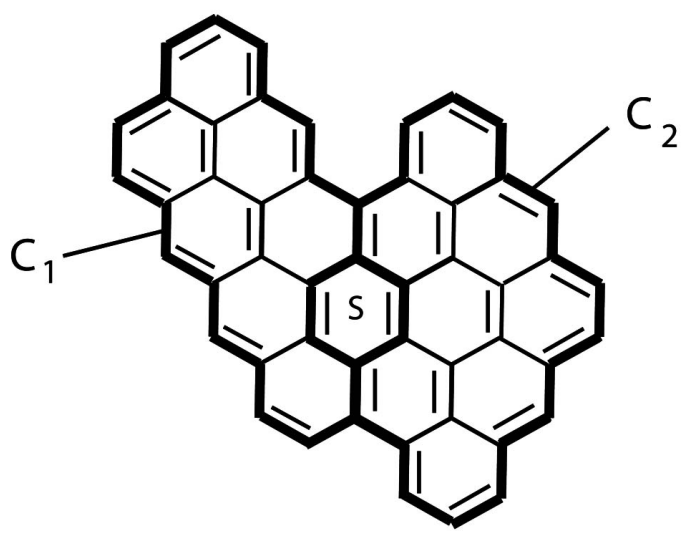

Fig. (18). $C_{1}$ and $C_{2}$ are two minimal $K_{i}$-conjugated circuits of a ring $s$.

Lemma 5.2 [108]. Let $K_{i}$ be a Kekulé structure of a benzenoid hydrocarbon $B$, and let $C_{1}$ and $C_{2}$ be two minimal $K_{i}$-conjugated circuits of a ring $s$ in $B$. Then,

$B\left[C_{1}\right]$ and $B\left[C_{2}\right]$ contain a unique ring $s$ in common;

any edge in $C_{1} \cap s\left(C_{2} \cap s\right)$ is not on $C_{2}\left(C_{1}\right)$, and any edge in $s-E\left(C_{1} \cap s\right)\left(s-E\left(C_{2} \cap s\right)\right)$ is on $C_{2}$ $\left(C_{1}\right)$;

each component in $C_{1} \cap C_{2}$ is an edge (which is a $K_{i}$-double bond); and

there are at most two minimal $K_{i}$-conjugated circuits of $s$ in $B$. 


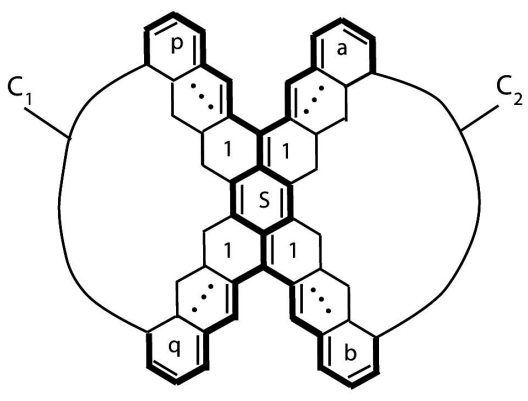

(1) $|\zeta|=\left|C \frac{2}{2}\right|^{4 n+2,}$ $p+q=a+b=n-1$, $p, q, a, b \geqslant 1$.

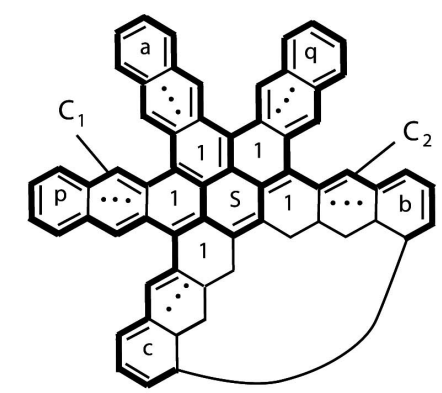

(3) $|G|=\mid C_{2} \neq 4 n+2$, $\mathrm{p}+\mathrm{q}=\mathrm{a}+\mathrm{b}+\mathrm{c}=\mathrm{n}-1$, $p, q, a, b, c \geqslant 1$.

Fig. (19). Four types of pairs of minimal conjugated circuits.

We call two minimal $K_{i}$-conjugated circuits of a ring $s$ in $B$ as a pair of minimal $K_{i}$-conjugated circuits of $s$. By Lemma 5.2, we can classify pairs of minimal conjugated circuits of a ring $\mathrm{s}$ in $\mathrm{B}$.

Theorem 5.12 [108]. Let $C_{1}$ and $C_{2}$ be a pair of minimal $K_{i}$-conjugated circuits of a ring $s$ in a benzenoid hydrocarbon $B$ for a Kekulé structure $K_{i}$ of $B$. Then $C_{1} \cup C_{2}$ belongs to one of the four types of pairs of minimal conjugated circuits as shown in Fig. (19).

Theorem 5.12 gives all possible configurations of a pair of minimal $K_{i}$-conjugated circuits $C_{1}$ and $C_{2}$ of a ring $s$ in $B$. We call these configurations $P M$-configurations w.r.t. the ring s, and $C_{1}$ and $C_{2}$ the mutually associated minimal conjugated circuits of $s$. Conversely, for a pair of mutually conjugated circuits $C_{1}$ and $C_{2}$ (i.e., $B-C_{1} \cup C_{2}$ has Kekulé structures) with a $P M$-configuration w.r.t. a ring $s, C_{1}$ and $C_{2}$ may have different sizes and are called mutually associated conjugated circuits of $S$.

Lemma 5.3 [108]. Let $C_{1}$ and $C_{2}$ be a pair of mutually associated conjugated circuits with the same size and with a $P M$-configuration w.r.t. a ring $s$ in a benzenoid hydrocarbon $B$. Then, for any Kekulé structure $K_{i^{\prime}}$ in $B-C_{1} \cup C_{2}$, there is exactly one Kekulé structure $K_{i}$ in $B$

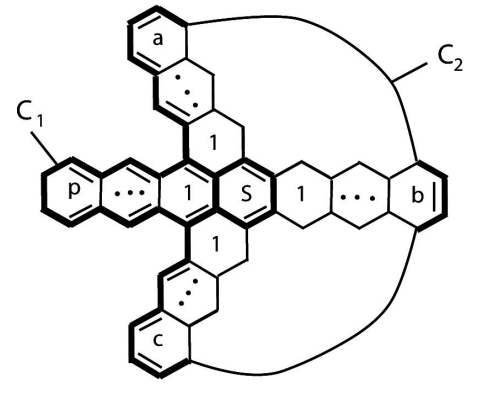

(2) $|G|=\left|C_{2}\right| 4 n+2$, $\mathrm{p}=\mathrm{a}+\mathrm{b}+\mathrm{c}=\mathrm{n}-1$, $p, a, b, c>1$.

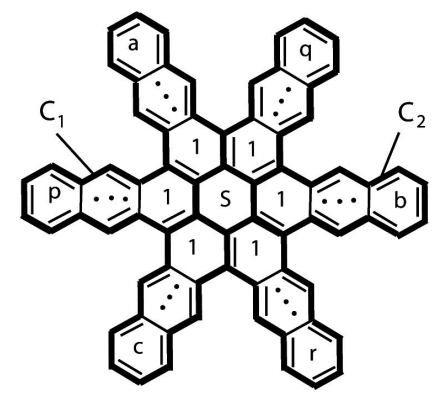

(4) $|G|=\mid C_{2}=4 n+2$, $p+q+r=a+b+c=n-1$, $p, q, r, a, b, c>1$. for which $K_{i^{\prime}} \subset K_{i}$ and both $C_{1}$ and $C_{2}$ are minimal $K_{i}$ conjugated circuits of $s$ in $B$.

By Lemma 5.3, we can also give a new definition of a pair of minimal conjugated circuits of a ring in $B$, which is independent of a Kekulé structure $K_{i}$ of $B$.

Definition 5.7 [108]. Two mutually conjugated circuits $C_{1}$ and $C_{2}$ in a benzenoid hydrocarbon $B$ are said to be a pair of minimal conjugated circuits of a ring $s$ in $B$, if $C_{1} \cup C_{2}$ has a $P M$-configuration w.r.t. the ring $s$, and $C_{1}$ and $C_{2}$ have the same size. We also say that $C_{1}$ and $C_{2}$ are mutually associated minimal conjugated circuits w.r.t. $s$.

Recall the construction of a minimal $K_{i}$-conjugated circuit $C$ of a ring $s$ in $B$ shown in Fig. (9). Let $B^{\prime}[C]$ be the subgraph of $B[C]$ induced by the hexagons labeled by $0,1,2, \cdots, a, b, c$, and $C^{\prime}$, the boundary of $B^{\prime}[C]$. Clearly, $C^{\prime}$ also has an $M$-configuration w.r.t. the ring $s$, and $\left|C^{\prime}\right|=|C|=4(a+b+c+1)+2=4 n+2 \quad\left(C \rightarrow R_{n}\right)$. We call $B^{\prime}[C]$ the underlying configuration of $B[C]$ and say that $C^{\prime}$ is the underlying circuit of $C$ and that $C^{\prime}$ has an underlying $M$-configuration w.r.t. $s$. For a minimal $K_{i}$ conjugated circuit $C$ of a ring $s$ in $B$, there is a unique circuit $C_{2} \neq C_{1^{\prime}}$ with an underlying $M$-configuration w.r.t. 
$s$ for which $B\left[C_{2}\right]$ contains as many hexagons as possible so that all interior edges of $B\left[C_{2}\right]$ are $K_{i}$-single bonds. The circuit $C_{2}$ is called the associated circuit of $C_{1}$ according to $K_{i}$. Particularly, if all edges of $s$ are not on the boundary of $B, C_{2}$ is called the nondegenerated associated circuit of $C_{1}$ (see Fig. 20). The edges $e_{a}, e_{b}$, and $e_{c}$ indicated in Fig. (20) are called the extreme edges of $C_{2}$; the sets of the interior edges of $B\left[C_{2}\right]$ parallel to $e_{a}, e_{b}$, and $e_{c}$ are, respectively, denoted by $E_{A^{\prime}}, E_{B^{\prime}}$, and $E_{C^{\prime}} ;$ and $E_{A}=E_{A^{\prime}} \cup\left\{e_{a}\right\}, E_{B}=E_{B^{\prime}} \cup\left\{e_{b}\right\}$, and $E_{C}=E_{C^{\prime}} \cup\left\{e_{c}\right\}$. Note that if any one of $e_{a}, e_{b}$, or $e_{c}$ is not on the boundary $b(B)$ of $B$, it must be a $K_{i}$-double bond. In the other case, it may be a $K_{i}$-single or $K_{i}$-double bond. The associated circuit $C_{2}$ of $C_{1}$ is different from an associated minimal $K_{i}$ conjugated circuit of $C_{1}$, since $C_{2}$ may be not $K_{i}$ conjugated and $\left|C_{2}\right|$ may be not equal to $\left|C_{1}\right|$.

Theorem 5.13 [108]. Let $K_{i}$ be a Kekulé structure of a benzenoid hydrocarbon $B ; C_{1}$, a minimal $K_{i}$-conjugated circuit of a ring $s$ in $B$; and $C_{2}$, the associated circuit of $C_{1}$ according to $K_{i}$. Then, if (1) an edge on $s$ lies on the boundary $b(B)$ of $B$, or (2) one of the extreme edges of $C_{2}$ lies on $b(B)$ and is a $K_{i}$-single bond, or (3) $C_{2}$ has greater size than $C_{1}, C_{1}$ is a unique minimal $K_{i}$-conjugated circuit of $s$ in $B$.

For a circuit $C$ with an $M$-configuration w.r.t. a ring $s$ in $B$ and the underlying circuit $C^{\prime}$ of $C$, there is a circuit $C^{*}$ with an $M$-configuration w.r.t. $s$ for which the underlying circuit of $C^{*}$ is just $C^{\prime}$, the interior of $C$ is contained in the interior of $C^{*}$, and $B\left[C^{*}\right]$ contains as many hexagons as possible. In the case in Fig. (21(1)), $C^{*}$ has the maximum $M$-configuration, called full $M$-configuration. In the case of Fig. (21(2)), $C^{*}$ is said to have a truncated
$M$-configuration. We call $C^{*}$ the characteristic circuit of $C^{\prime}$ and $C$. For a minimal $K_{i}$-conjugated circuit $C_{1}$ of a ring $s$ and the associated circuit $C_{2}$ of $C_{1}$ according to $K_{i}$, a characteristic circuit $C_{2}^{*}$ of $C_{2}$ such that $C_{1} \cup C_{2}^{*}$ has a $P M$-configuration w.r.t. $s$ is also called the associated characteristic circuit of $C_{1}$. If a characteristic circuit $C^{*}$ of $C$ has a truncated $M$-configuration, for the edges $f_{1}, f_{2}, \cdots, f_{t}$ on $C^{*}$ which lie on the boundary $b(B)$ of $B$ and whose end vertices have degree three in $B\left[C^{*}\right]$, let $F_{i}$ denote the set of the edges in $B\left[C^{*}\right]$ which contain $f_{i}$ and are intersected by a same line segment $L_{i}$ (see Fig. 21(2)). We call $F_{i}, i=1,2, \cdots, t$, the characteristic edge sets of $B\left[C^{*}\right]$.

Theorem 5.14 [108]. Let $C_{1}$ be a minimal $K_{i}$ conjugated circuit of a ring $s$ of a benzenoid hydrocarbon $B$ for a Kekulé structure $K_{i}$ of $B ; C_{2}$, the nondegenerated associated circuit of $C_{1}$ according to $K_{i}$ with $\left|C_{2}\right| \leq\left|C_{l}\right|$; and $C_{2}^{*}$, the associated characteristic circuit of $C_{1}$. Then, $C_{1}$ is a unique minimal $K_{i}$-conjugated circuit of $s$ if and only if either an extreme edge of $C_{2}$ lies on the boundary of $B$ and is a $K_{i}$-single bond or $C_{2}^{*}$ has a truncated $M$-configuration and there is a characteristic edge set $F_{j}$ of $B\left[C_{2}^{*}\right]$ such that each edge in $F_{j}$ is a $K_{i}$-double bond.

By Theorems 5.3 and 5.4, we need only to consider the benzenoid hydrocarbons with no fixed bond. To calculate $R_{s}(B)$, it is enough to give a method to determine the coefficient $r_{n}(s)$ of the term $R_{n}$ in $R_{s}(B)$ for $n \geq 1$.

From a Kekulé structure $K_{i}$ and a minimal $K_{i}$ conjugated circuit $C$ of a ring $s$ in $B$, we have introduced the concepts of the associated circuit and the associated characteristic circuit of $C$, according to $K_{i}$. Now, we need

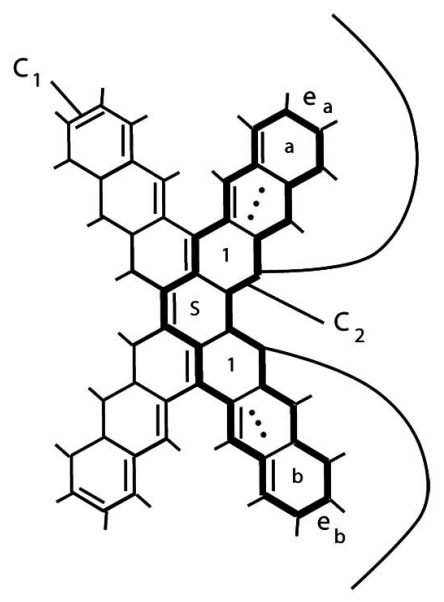

(1) non-degenerated

(2) degenerated

(3) degenerated

Fig. (20). The associated circuit $C_{2}$ of a minimal $K_{i}$-conjugated circuit $C_{1}$ of a ring $s$ in $B$ according to $K_{i}$. 

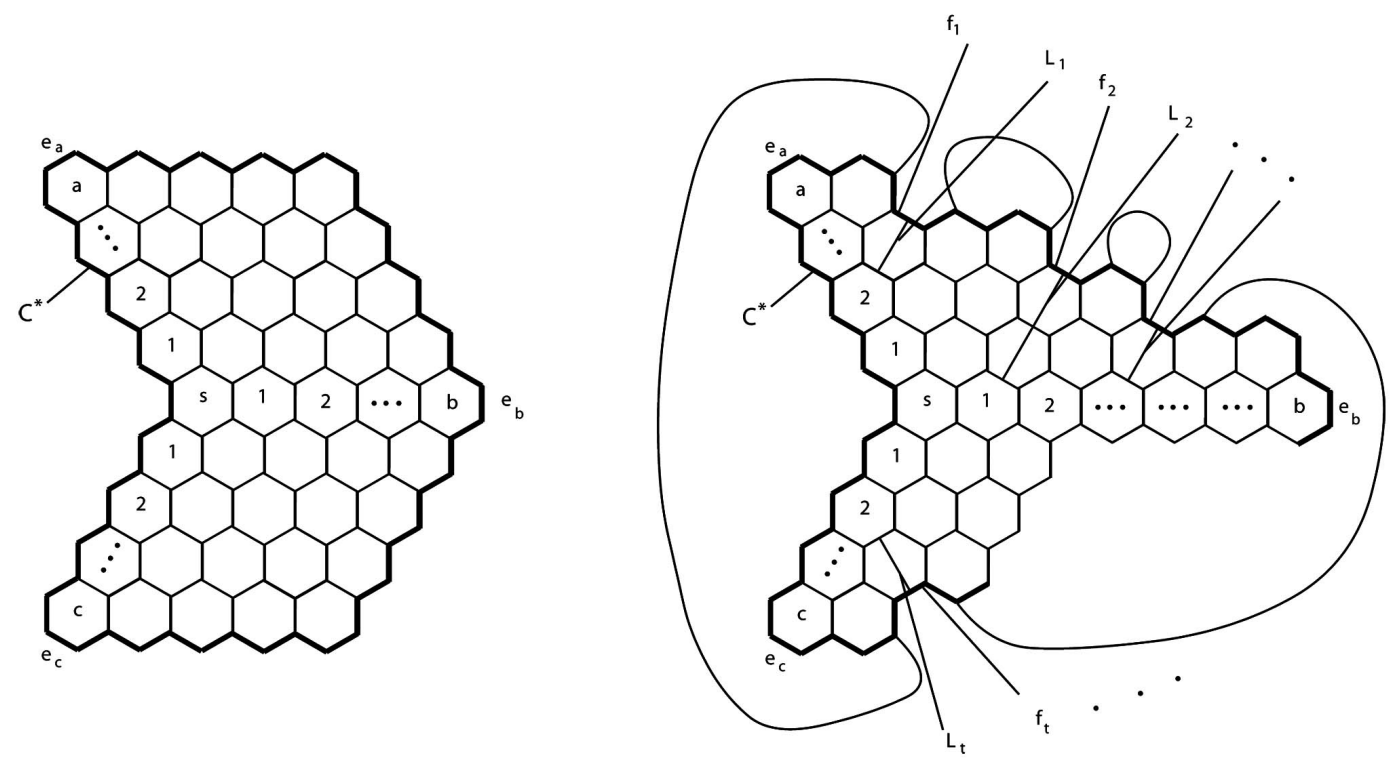

Fig. (21). (1) A characteristic circuit $C^{*}$ of $C$ with a full $M$-configuration. (2) A characteristic circuit $C^{*}$ of $C$ with a truncated $M$ configuration, where $f_{1}, f_{2}, \cdots, f_{t}$ lie on the boundary of $B$ and their end vertices have degree three in $B\left[C^{*}\right]$.

to generalize the concepts so that they are independent of a Kekulé structure of $B$.

Definition 5.8 [108]. For a pair of minimal conjugated circuits $C_{1}$ and $C_{2}$ of a ring $s$ in a benzenoid hydrocarbon $B$ and their underlying circuits $C_{1^{\prime}}$ and $C_{2^{\prime}}, C_{1^{\prime}} \cup C_{2^{\prime}}$ is said to have an underlying $P M$-configuration w.r.t. $s$. For a minimal conjugated circuit $C_{1}$ of $s$ and its underlying circuit $C_{1^{\prime}}$, a circuit $C_{2^{\prime}}$ is said to an nondegenerated associated circuit of $C_{1}$ and $C_{1^{\prime}}$, if $C_{1} \cup C_{2^{\prime}}$ has a $P M$ configuration w.r.t. $s, C_{1^{\prime}} \cup C_{2^{\prime}}$ has an underlying $P M$ configuration w.r.t. $s$, and any edge of $s$ is not on the boundary of $B$. A characteristic circuit $C_{2}^{*}$ of $C_{2^{\prime}}$ is said to be an associated characteristic circuit of $C_{1}$ and $C_{1^{\prime}}$ if $C_{1} \cup C_{2}^{*}$ has a $P M$-configuration w.r.t. $s$.

For a minimal conjugated circuit $C_{1}$ of a ring $s$ in $B$, let $A\left(C_{1}\right)=\left\{C_{2} \mid C_{2}\right.$ is a nondegenerated associated circuit of $\left.C_{1}\right\}$,

$A_{1}\left(C_{1}\right)=\left\{C_{2} \mid C_{2} \in A\left(C_{1}\right)\right.$, and $\left.\left|C_{2}\right|<\left|C_{1}\right|\right\}$,

$A_{2}\left(C_{1}\right)=\left\{C_{2} \mid C_{2} \in A\left(C_{1}\right)\right.$, and $\left.\left|C_{2}\right|=\left|C_{1}\right|\right\}$,

$A^{*}\left(C_{1}\right)=\left\{C_{2}^{*} \mid C_{2}^{*}\right.$ is an associated characteristic circuit of $\left.C_{1}\right\}$,

$A_{1}^{*}\left(C_{1}\right)=\left\{C_{2}^{*}\left|C_{2}^{*} \in A^{*}\left(C_{1}\right),\right| C_{2}^{*}|<| C_{1} \mid, \quad\right.$ and $C_{2}^{*}$ has a truncated $M$-configuration $\}$,

$A_{2}^{*}\left(C_{1}\right)=\left\{C_{2}^{*}\left|C_{2}^{*} \in A^{*}\left(C_{1}\right),\right| C_{2}^{*}|=| C_{1} \mid, \quad\right.$ and $C_{2}^{*}$ has a truncated $M$-configuration $\}$.

For calculation of $R_{s}(B)$, the pairs of circuits with underlying $P M$-configurations play a key role. Fig. (22) shows four types of pairs of circuits with underlying $P M$ configurations w.r.t. a ring $s$.

For a pair of circuits $C_{1}$ and $C_{2}$ in $B$ with an underlying $P M$-configuration, we denote by $C_{1}^{*}$ (respectively, $C_{2}^{*}$ ) the characteristic circuit of $C_{1}$ (respectively, $C_{2}$ ) which belongs to $A^{*}\left(C_{2}\right)$ [respectively, $A^{*}\left(C_{1}\right)$ ], where the circuit $C_{1}$ (respectively, $\left.C_{2}\right)$ is said to be of type $i \quad(i \in\{1,2,3,4\})$, if the pair of circuits $C_{1} \cup C_{2}$ is of type $i$. A minimal conjugated circuit $C$ of a ring $s$ is said to be of type $i$, if the underlying circuit of $C$ is isomorphic to one of circuits $C_{1}$ and $C_{2}$ of type $i$. For convenience, we introduce the following notations:

$C_{n}^{(i)}(s)=\left\{C \mid C\right.$ is a circuit in a pair of circuits $C_{1}$ and $C_{2}$, with an underlying $P$-configuration w.r.t. a ring $s$ in $B$ which are of type $i$, and $|C|=4 n+2\}$.

$r_{n}^{(i)}(s)$ : the number of all the circuits denoted by $R_{n}$ in $R_{s}(B)$ which are of type $i, i=1,2,3,4$.

$K\left(B\left[C_{1}, C_{2}\right]\right)$ : the number of the Kekule structures of $B$ for which all edges in $C_{1} \cap C_{2}$ and all extreme edges $\left(e_{a}, \cdots, e_{p}, \cdots\right)$ of $C_{1}$, and $C_{2}$ are double bonds, and all interior edges of $B\left[C_{1}\right]$ and $B\left[C_{2}\right]$ are single bonds.

$K\left(B\left[C_{1}, C_{2}^{*}\right]\right)$ : the number of the Kekule structures of $B$ for which all extreme edges of $C_{1}$ and $C_{2}$, and all edges in $C_{1} \cap C_{2}$ and a characteristic edge set of $B\left[C_{2}^{*}\right]$ are double bonds, and all interior edges of $B\left[C_{1}\right]$ and $B\left[C_{2}\right]$ are single bonds.

$K\left(B\left[C_{i}, E_{1}, \bar{E}_{2}\right]\right)$ : the number of the Kekule structures of $B$ for which all extreme edges of $C_{i}$, and the edges in 


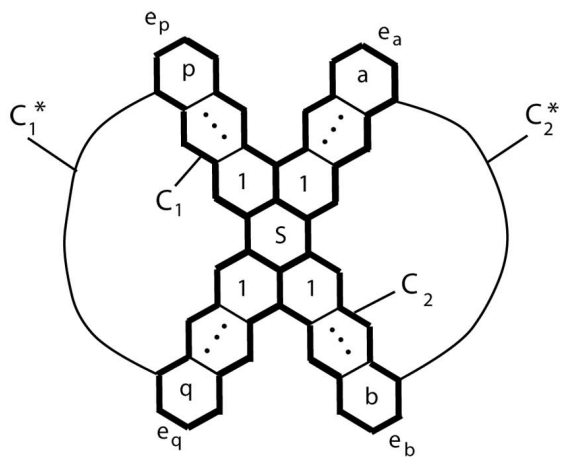

(1) Type 1, a,b,p,q>1,

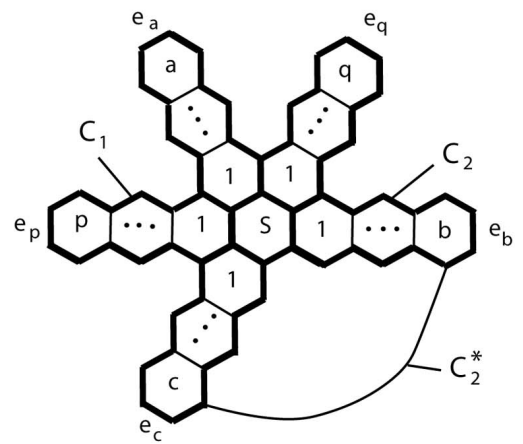

(3) Type 3, a,b,c,p,q>1,

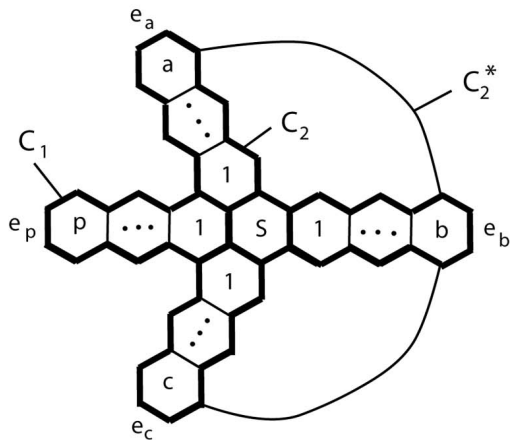

(2) Type 2, a,b,c,p>1,

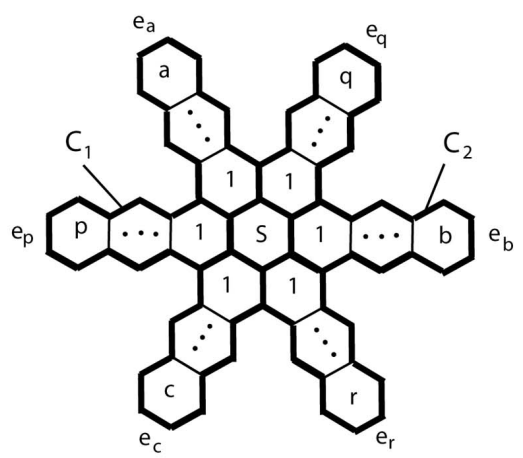

(4) Type 4, a,b,c,p,q,r>1,

Fig. (22). Four types of pairs of circuits with underlying $P M$-configurations w.r.t. a ring $s$ in $B$, where $C_{2}^{*} \in A^{*}\left(C_{1}\right), C_{1}^{*} \in A^{*}\left(C_{2}\right)$.

$E\left(C_{1} \cap C_{2}\right) \cup E_{l}$ are double bonds, and all interior edges of $B\left[C_{i}\right]$ and the edges in $E_{2}$ are single bonds.

$K\left(B\left[C_{i}, E_{1}, \bar{E}_{2}\right]\right):$ the number of the Kekule structures of $B$ for which all extreme edges of $C$, and the edges in $E\left(C_{1} \cap C_{2}\right) \cup E_{l}$ and a characteristic edge set of $C_{i}^{*}$ are double bonds, and all interior edges of $B\left[C_{i}\right]$ and the edges in $E_{2}$ are single bonds.

$K\left(B\left[C_{1}, C_{2}, E_{1}, \bar{E}_{2}\right]\right)$ : the number of the Kekule structures of $B$ for which the edges in $E\left(C_{1} \cap C_{2}\right) \cup E_{l}$ and all extreme edges of $C_{1}$ and $C_{2}$ are double bonds, and the edges in $E_{2}$ and all interior edges of $B\left[C_{1}\right]$ and $B\left[C_{2}\right]$ are single bonds.

$K\left(B\left[C_{i}, \phi\right]\right)$ : the number of the Kekule structures of $B$ for which $A\left(C_{i}\right)=\phi$, and $C_{i} \cap C_{i}^{*}$ is an alternating path (or circuit) in double and single bonds such that the extreme edges of $C_{i}$ are double bonds.

$E_{A}^{*}$ : the edge set $E_{A}$ for a circuit $C_{2} \in A\left(C_{1}\right)$ satisfying that, if $C_{1} \cup C_{2}$ is of type 1 (respectively, types 2, 3, 4), $\left|E_{A}^{*}\right|=\left|E_{A}\right|=n-1$, or $\left|E_{A}^{*}\right|<n-1$ but $e_{a}$ is on $b(B)$ [respectively, $\left|E_{A}^{*}\right|=\left|E_{A}\right|=n-2$, or $\left|E_{A}^{*}\right|<n-2$, but $e_{a}$ is on $b(B)]$.
$E_{A B}^{*}$ : the edge set $E_{B}$ for a circuit $C_{2} \in A\left(C_{1}\right)$ satisfying that, if .. is of type 1 (respectively, types 2, 3, 4), $\left|E_{A}\right| \leq n-2 \quad$ and $\quad\left|E_{A B}^{*}\right|=\left|E_{B}\right|=n-1-\left|E_{A}\right|, \quad$ or $\left|E_{A}\right|+\left|E_{A B}^{*}\right|<n-1$ but $e_{b}$ is on $b(B)$ (respectively, $\left|E_{A}\right| \leq n-3$ and $\left|E_{A}\right|+\left|E_{A B}^{*}\right|=n-2$, or $\left|E_{A}\right|+\left|E_{A B}\right|<n-2$ but $e_{b}$ is on $b(B)$ ).

$E_{A B C}^{*}$ : the edge set $E_{C}$ for a circuit $C_{2} \in A\left(C_{1}\right)$ satisfying that $\left|E_{A}\right|+\left|E_{B}\right| \leq n-2$ and $\left|E_{A}\right|+\left|E_{B}\right|+\left|E_{A B C}^{*}\right|=n-1$, or $\left|E_{A}\right|+\left|E_{B}\right|+\left|E_{A B C}\right|<n-1$ but $e_{c}$, is on $b(B)$, where $C_{1} \cup C_{2}$ is of types $2,3,4$.

Similarly, we can define $K\left(B\left[C_{2}, C_{1}^{\prime \prime}\right]\right), E_{P}^{*}, E_{P Q}^{*}$, and $E_{P Q R}^{*}$. Note that $B\left[C_{1}, C_{2}\right], B\left[C_{1}, C_{2}^{*}\right], B\left[C_{i}, E_{1}, \bar{E}_{2}\right], B\left[C_{i}^{*}, E_{1}, \bar{E}_{2}\right], \cdots$, denote the graphs obtained from $B$ by labeling some edges as double or single bonds.

Lemma 5.4 [108]. Let $C_{1}$ and $C_{2}$ be a pair of circuits with an underlying $P M$-configuration w.r.t. a ring $S$ in a benzenoid hydrocarbon $B$, and $C_{2}^{*}$ the associated characteristic circuit of $C_{1}$. Then, 
$K\left(B\left[C_{1}, C_{2}^{*}\right]\right)=\sum_{1 \leq i \leq t} K\left(B\left[C_{1}, C_{2}, F_{i}\right]\right)-\sum_{1 \leq i<j \leq t}$

$K\left(B\left[C_{1}, C_{2}, F_{i} \cup F_{j}\right]\right)$

$+\sum_{1 \leq i<j<k \leq t} K\left(B\left[C_{1}, C_{2}, F_{i} \cup F_{j} \cup F_{k}\right]\right)-\cdots+(-1)^{t-1}$

$K\left(B\left[C_{1}, C_{2}, \cup_{i=1}^{t} F_{i}\right]\right)$

$K\left(B\left[C_{2}^{*}, E_{1}, \bar{E}_{2}\right]\right)=\sum_{1 \leq i \leq t} K\left(B\left[C_{2}, F_{i} \cup E_{1}, \bar{E}_{2}\right]\right)-$

$\sum_{1 \leq i<j \leq t} K\left(B\left[C_{2}, F_{i} \cup F_{j} \cup E_{1}, \bar{E}_{2}\right]\right)$

$+\cdots+(-1)^{t-1} K\left(B\left[C_{2}, E_{1} \cup\left(\cup_{i=1}^{t} F_{i}\right), \bar{E}_{2}\right]\right)$,

where $F_{1}, F_{2}, \cdots, F_{t}$ are all characteristic edge sets of $C_{2}^{*}$.

Now we can give a method for calculating $r_{n}^{(i)}(s)$. Consequently, $r_{n}(s)=\sum_{i=1}^{4} r_{n}^{(i)}(s)$.

Theorem 5.15 [108]. Let $B$ be a benzenoid hydrocarbon with no fixed bond, and $s$, a ring of $B$. Then,

$$
\text { (i) } \begin{aligned}
r_{n}^{(1)}(s) & =\sum_{C_{1} \in C_{n}^{(1)}(s), A\left(C_{1}\right) \neq \phi}\left\{K\left(B\left[C_{1}, \overline{E_{A}^{*}}\right]\right)-K\left(B\left[C_{1}^{*}, \overline{E_{A}^{*}}\right]\right)\right. \\
& +\sum_{1 \leq a \leq n-2}\left[K\left(B\left[C_{1}, e_{a}, \overline{E_{A}^{\prime} \cup E_{A B}^{*}}\right]\right)-K\left(B\left[C_{1}^{*}, e_{a}, \overline{E_{A}^{\prime} \cup E_{A B}^{*}}\right]\right)\right] \\
& \left.+\sum_{C_{2}^{*} \in A_{1}^{*}\left(C_{1}\right)} K\left(B\left[C_{1}, C_{2}^{*}\right]\right)+\sum_{C_{2} \in A_{2}\left(C_{1}\right)} K\left(B\left[C_{1}, C_{2}\right]\right)\right\} \\
& +\sum_{C_{2} \in C_{n}^{(1)}(s), A\left(C_{2}\right) \neq \phi}\left\{K\left(B\left[C_{2}, \overline{E_{P}^{*}}\right]\right)-K\left(B\left[C_{2}^{*}, \overline{E_{P}^{*}}\right]\right)\right. \\
& +\sum_{1 \leq p \leq n-2}\left[K\left(B\left[C_{2}, e, \overline{E_{P}^{\prime} \cup E_{P Q}^{*}}\right]\right)-K\left(B\left[B\left[C_{2}^{*}, e_{p}, \overline{E_{P}^{\prime} \cup E_{P Q}^{*}}\right]\right)\right]\right. \\
& \left.+\sum_{C_{1}^{*} \in A_{1}^{*}\left(C_{2}\right)} K\left(B\left[C_{2}, C_{1}^{*}\right]\right)\right\} \\
& +\sum_{C_{1} \in C_{n}^{(1)}(s), A\left(C_{1}\right)=\phi}\left[K\left(B\left[C_{1}, \phi\right]\right)-K\left(B\left[C_{1}^{*}, \phi\right]\right)\right] \\
& +\sum_{C_{2} \in C_{n}^{(1)}(s), A\left(C_{2}\right)=\phi}\left[K\left(B\left[C_{2}, A\right]\right)-K\left(B\left[C_{2}^{*}, \phi\right]\right)\right],
\end{aligned}
$$

where $C_{1}\left(\in C_{n}^{(1)}(s)\right)$ and $C_{2}\left(\in C_{n}^{(1)}(s)\right)$ are taken for three possible positions of $C_{1} \cup C_{2}$ in B.

$$
\text { (ii) } \begin{aligned}
r_{n}^{(2)}(s)= & \sum_{C_{1} \in C_{n}^{(2)}(s), A\left(C_{1}\right) \neq \phi}\left\{K\left(B\left[C_{1}, \overline{E_{A}^{*}}\right]\right)+\sum_{1 \leq a \leq n-3} K\left(B\left[C_{1}, e_{a}, \overline{E_{A}^{\prime} \cup E_{A B}^{*}}\right]\right)\right. \\
& +\sum_{2 \leq a+b \leq n-2, a, b \geq 1} K\left(B\left[C_{1},\left\{e_{a}, e_{b}\right\}, \overline{E_{A}^{\prime} \cup E_{B}^{\prime} \cup E_{A B C}^{*}}\right]\right) \\
& \left.+\sum_{C_{2} \in A_{2}\left(C_{1}\right)} K\left(B\left[C_{1}, C_{2}\right]\right)+\sum_{C_{2}^{*} \in A_{1}^{*}\left(C_{1}\right)} K\left(B\left[C_{1}, C_{2}^{*}\right]\right)\right\} \\
& +\sum_{C_{2} \in C_{n}^{(2)}(s), A\left(C_{2}\right) \neq \phi} K\left(B\left[C_{2}, \overline{E_{p}^{*}}\right]\right)+\sum_{C_{1} \in C_{n}^{(2)}(s), A\left(C_{1}\right)=\phi}\left[K\left(B\left[C_{1}, \phi\right]\right),\right. \\
& \left.\left.-K\left(B\left[C_{1}^{*}, \phi\right]\right)\right]+\sum_{C_{2} \in C_{n}^{(2)}(s), A\left(C_{2}\right)=\phi}\left[K\left(B\left[C_{2}, \phi\right]\right)-K\left(B\left[C_{2}^{*}, \phi\right]\right]\right)\right]
\end{aligned}
$$

where $C_{1}$ and $C_{2}$ are taken for six possible positions of $C_{1} \cup C_{2}$ in $\mathrm{B}$.

$$
\text { (iii) } \begin{aligned}
r_{n}^{(3)}(s)= & \sum_{C_{1} \in C_{n}^{(3)(s), A\left(C_{1}\right) \neq \phi}}\left\{K\left(B\left[C_{1}, \overline{E_{A}^{*}}\right]\right)+\sum_{1 \leq a \leq n-3} K\left(B\left[C_{1}, e_{a}, \overline{E_{A}^{\prime} \cup E_{A B}^{*}}\right]\right)\right. \\
& +\sum_{2 \leq a+b \leq n-2, a, b \geq 1} K\left(B\left[C_{1},\left\{e_{a}, e_{b}\right\}, \overline{E_{A}^{\prime} \cup E_{B}^{\prime} \cup E_{A B C}^{*}}\right]\right) \\
& \left.+\sum_{C_{2} \in A_{2}\left(C_{1}\right)} K\left(B\left[C_{1}, C_{2}\right]\right)+\sum_{C_{2}^{*} \in \epsilon_{1}^{*}\left(C_{1}\right)} K\left(B\left[C_{1}, C_{2}^{*}\right]\right)\right\} \\
& +\sum_{C_{2} \in C_{n}^{(3)}(s), A\left(C_{2}\right) \neq \phi}\left\{K\left(B\left[C_{2}, \overline{E_{P}^{*}}\right]\right)+\sum_{1 \leq p \leq n-2}\left[K\left(B\left[C_{2}, e_{p}, \overline{E_{P}^{\prime} \cup E_{P Q}^{*}}\right]\right)\right.\right. \\
& \left.-K\left(B\left[C_{2}^{*}, e_{p}, E_{P}^{\prime} \cup E_{P Q}^{*}\right]\right)\right\}+\sum_{C_{1} \in c_{n}^{(3)}(s), A\left(C_{1}\right)=\phi}\left[K\left(B\left[C_{1}, \phi\right]\right)\right. \\
& \left.\left.-K\left(B\left[C_{1}^{*}, \phi\right]\right]\right)\right]+\sum_{C_{2} \in C_{n}^{(3)}(s), A\left(C_{2}\right)=\phi}\left[K\left(B\left[C_{2}, \phi\right]\right)-D\left(B\left[C_{2}^{*}, \phi\right]\right]\right],
\end{aligned}
$$

where $C_{1}$ and $C_{2}$ are taken for six possible positions of $C_{1} \cup C_{2}$ in B.

$$
\text { (iv) } \begin{aligned}
r_{n}^{(4)}(s)= & \sum_{C_{1} \in C_{n}^{(4)}(s), A\left(C_{1}\right) \neq \phi}\left\{K\left(B\left[C_{1}, \overline{E_{A}^{*}}\right]\right)+\sum_{1 \leq a \leq n-3} K\left(B\left[C_{1}, e_{a}, \overline{E_{A}^{\prime} \cup E_{A B}^{*}}\right]\right)\right. \\
& +\sum_{2 \leq a+b \leq n-2, a, a, b 1} K\left(B\left[C_{1},\left\{e_{a}, e_{b}\right\}, \overline{E_{A}^{\prime} \cup E_{B}^{\prime} \cup E_{A B C}^{*}}\right]\right) \\
& \left.+\sum_{C_{2} \in A_{2}\left(C_{1}\right)} K\left(B\left[C_{1}, C_{2}\right]\right)\right\}+\sum_{C_{2} \in C_{n}^{(4)}(s), A\left(C_{2}\right) \neq \phi}\left\{K\left(B\left[C_{2}, \overline{E_{P}^{*}}\right]\right)\right. \\
& +\sum_{1 \leq p \leq n-3}\left[K\left(B\left[C_{2}, e_{p}, \overline{E_{P}^{\prime} \cup E_{P Q}^{*}}\right]\right)\right. \\
& +\sum_{2 \leq p+q \leq n-2, p, q \geq 1} K\left(B\left[C_{2},\left\{e_{p}, e_{q}\right\}, \overline{E_{P}^{\prime} \cup E_{Q}^{\prime} \cup E_{P Q R}^{*}}\right]\right) \\
& +\sum_{\left.C_{1} \in C_{n}^{(4)}(s), A C_{1}\right)=\phi}\left[K\left(B\left[C_{1}, \phi\right]\right)-K\left(B\left[C_{1}^{*}, \phi\right]\right)\right] \\
& +\sum_{C_{2} \in C_{n}^{(4)(s), A\left(C_{2}\right)=\phi}}\left[K\left(B\left[C_{2}, \phi\right]\right)-K\left(B\left[C_{2}^{*}, \phi\right]\right)\right],
\end{aligned}
$$

where $C_{1}$ and $C_{2}$ are taken for one possible position of $C_{1} \cup C_{2}$ in $\mathrm{B}$.

Note that the contribution of $R(B)$ to the resonance energy of $B$ is mostly due to the conjugated circuits denoted by $R_{1}, R_{2}, R_{3}$. The expressions for $r_{1}(s), r_{2}(s)$ and $r_{3}(s)$ can be obtained from Theorems 5.12 and 5.15 .

Corollary 5.17 [108]. Let $B$ be a benzenoid hydrocarbon with no fixed bond and $S$ a ring in $B$. Then

(i) $r_{1}(s)=2 K(B-s)$,

$r_{2}(s)=\sum_{s_{i} \cap s z \phi} K\left(B-s \cup s_{i}\right)$, where $s_{i}$ is a hexagon adjacent to $S$,

$$
\begin{aligned}
& r_{3}^{(1)}(s)=\sum_{C_{1} \in C_{3}^{(1)}(s), A\left(C_{1}\right) \neq \phi}\left[K\left(B-C_{1}^{*}\right)+K\left(B-C_{2}^{*}-e_{p}\right)+\right. \\
& \left.K\left(B-C_{2}^{*}-V\left(e_{p}\right)-e_{q}\right)\right] \\
& +\sum_{C_{1} \in C_{3}^{(1)}(s), A\left(C_{1}\right)=\phi, C_{1} \in C_{1}^{*}} K\left(B-C_{1}^{*}\right)+ \\
& \sum_{C_{2} \in C_{3}^{(1)}(s), A\left(C_{2}\right)=\phi, C_{2} \neq C_{2}^{*}} K\left(B-C_{2}^{*}\right),
\end{aligned}
$$

where $C_{1}$ and $C_{2}$ are taken for three possible positions of $C_{1} \cup C_{2}$ in $B$,

$0.8 \mathrm{~cm} r_{3}^{(2)}(s)=\sum_{C_{1} \in C_{3}^{(2)}(s)} K\left(B-C_{1}\right)$,

$0.8 \mathrm{~cm} r_{3}^{(3)}(s)=\sum_{C_{1} \in C_{3}^{(3)}(s)} K\left(B-C_{1}\right)$,

$0.8 \mathrm{~cm} r_{3}^{(4)}(s)=0$.

\section{$6 k$-RESONANCE IN CHEMICAL GRAPHS AND $k$ - CYCLE RESONANT GRAPHS}

Let $G$ be a benzenoid system, open-ended carbon nanotube (tubule), toroidal or Klein-bottle polyhex. We say $G$ is $k$-resonant if, for $1 \leq t \leq k$, any $t$ disjoint hexagons of $G$ are mutually resonant, that is, there is a Kekulé structure (or perfect matching) $K$ of $G$ such that each of the $k$ hexagons is a $K$-alternating hexagon. A connected graph $G$ is said to be $k$-cycle resonant if, for $1 \leq t \leq k$, 
any $t$ disjoint cycles in $G$ are mutually resonant. The concept of $k$-resonant graphs is closely related to Clar's aromatic sextet theory, and the concept of $k$-cycle resonant graphs is a natural generalization of $k$-resonant graphs.

Some necessary and sufficient conditions for a benzenoid system or a tubule or a toroidal polyhex (resp. a graph) to be $k$-resonant (resp. $k$-cycle resonant) have been established. A survey on investigations of k-resonant benzenoid systems, $k$-resonant tubules, $k$-resonant toroidal polyhexes, and $k$ cycle resonant graphs was given in [109]. For recent works on $k$-resonance, the reader is referred to [69], and [110$115]$.

\section{CONFLICT OF INTEREST}

There is no conflict of interest.

\section{ACKNOWLEDGEMENT}

This work is supported by Grants from the National Natural Science Foundation of China (No. 10830110). We would like to thank Professors Alexandru Balaban, Zhibo Chen, and referees for their helpful suggestions.

\section{ABBREVIATIONS}

$\begin{array}{lll}\mathrm{VB} & = & \text { Valence-bond } \\ \mathrm{RE} & = & \text { Resonance energy } \\ \mathrm{TRE} & = & \text { Topological resonance energy } \\ \mathrm{DRE} & \text { Dewar resonance energy } \\ \begin{array}{l}\mathrm{LM} \text {-conjugated } \\ \text { circuit }\end{array} & \begin{array}{l}\text { Linear independent and minimal } \\ \text { conjugated circuit }\end{array} \\ \begin{array}{l}\text { LM-conjugated } \\ \text { circuit }\end{array} & \begin{array}{l}\text { Linear independent and minimal } \\ \text { polynomial (LMC-expression) }\end{array}\end{array}$

\begin{tabular}{|c|c|c|}
\hline $\begin{array}{l}\text { LMCC- } \\
\text { polynomial }\end{array}$ & $=$ & LM-conjugated circuit polynomial \\
\hline $\mathrm{BH}$ & $=$ & Benzenoid hydrocarbon \\
\hline $\mathrm{BHF}$ & $=$ & Benzenoid hydrocarbon-fragment \\
\hline $\mathrm{GBH}$ & $=$ & Generalized benzenoid hydrocarbon \\
\hline cata-BH & $=$ & Catacondensed benzenoid hydrocarbon \\
\hline peri-BH & $=$ & Pericondensed benzenoid hydrocarbon \\
\hline $\begin{array}{l}\text { M- } \\
\text { configuration }\end{array}$ & $=$ & Minimal configuration \\
\hline $\begin{array}{l}\text { M- } \\
\text { onfiguration }\end{array}$ & $=$ & $\begin{array}{l}\text { Configurations of a pair of minimal } \\
\text { conjugated circuits }\end{array}$ \\
\hline
\end{tabular}

\section{REFERENCES}

[1] Klein, D.J. Semiempirical valence bond views for benzenoid hydrocarbons. In: Advences in the Theory of Benzenoid Hydrocarbons; Springer-Verlag: Berlin, 1990, pp. 57-83.

[2] Wheland G.W. Resonance in Organic Chemistry; Wiley: New York, 1955; pp. 279-290.

[3] Armit, J.W.; Robinson, R. Polynuclear heterocyclic aromatic types. Part II. Some anhydronium bases. J. Chem. Soc., Trans., 1925, 1604-1618.
[4] Hückel, E. Quantentheoretische Beiträge zum Benzolproblem I. Die Elektronenkonfiguration des Benzols und verwandter Verbindungen. Z. Phys., 1931, 70, 204-286.

[5] Hückel, E. Quanstentheoretische Beiträge zum Benzolproblem II. Quantentheorie der induzierten Polaritäten. Z. Phys., 1931, 72, 310337.

[6] Hückel, E. Quantentheoretische Beiträge zum Problem der aromatischen und ungesättigten Verbindungen. III. Z. Phys., 1932, 76, 628-648.

[7] Gutman, I. Topological properties of benzenoid molecules. Bull. Soc. Chem. Beograd, 1982, 47, 453-472.

[8] Clar, E. The Aromatic Sextet; Wiley: London, 1972.

[9] Vijayalakshmi, K.P.; Suresh, C.H. Pictorial representation and validation of Clar's aromatic sextet theory using molecular electrostatic potentials. New J. Chem., 2010, 34, 2132-2138.

[10] Zhang, F.; Chen, R.; Guo, X.; Gutman, I. An invariant of the Kekulé structures of benzenoid hydrocarbons, J. Serb. Chem. Soc., 1986, 51, 537-543.

[11] Zhang, F.; Li, X. The Clar formula of a class of hexagonal systems. MATCH Commun. Math. Comput. Chem., 1989, 24, 333-347.

[12] Zhang, H. The Clar formula of regular $t$-tier strip benzenoid systems. System Sci. and Math. Sci., 1995, 8, 327-337.

[13] Zhang, H. The Clar formula of hexagonal polyexes. J. Xinjiang Univ. Nat. Sci., 1995, 12, 1-9.

[14] Salema, K.; Gutman, I. Clar number of hexagonal chains. Chem. Phys. Lett., 2004, 394, 283-286.

[15] Klavžar, S.; Žigert, P.; Gutman, I. Clar number of catacondensed benzenoid hydrocarbons. J. Mol. Struct. (Theochem), 2002, 586, 235-240.

[16] Balaban, A.T. Clar formulas: how to draw and how not to draw formulas of polycyclic aromatic hydrocarbons. Polyc. Arom. Comp., 2004, 24, 83-89.

[17] Hansen, P.; Zheng, M. Upper bounds for the Clar number of a benzenoid hydrocarbon. J. Chem. Soc. Faraday Trans., 1992, 88, 1621-1625

[18] Hansen, P.; Zheng, M. The Clar number of a benzenoid hydrocarbon and linear programming. J. Math. Chem., 1994, 15, 93-107.

[19] Abeledo, H.; Atkinson, G. Unimodularity of the Clar number problem. Linear Alg. Appl., 2007, 420, 441-448.

[20] Hosoya, H.; Yamaguchi, T. Sextet polynomial. A new enumeration and proof technique for the resonance theory applied to the aromatic hydrocarbons. Tetrahedron Lett., 1975, 4659-4662.

[21] El-Basil, S.; Randić, M. Clar valence structures of benzenoid hydrocarbons. J. Chem. Soc. Faraday Trans., 1988, 2, 1875-1887.

[22] Zhang, H.; Zhang, F. The Clar covering polynomial of hexagonal systems I. Discrete Appl. Math., 1996, 69, 147-167.

[23] Randić, M. Conjugated circuits and resonance energies of benzenoid hydrocarbons. Chem. Phys. Lett., 1976, 38, 68-70.

[24] Randić, M. Aromaticity and conjugation. J. Amer. Chem. Soc., 1977, 99, 444-450.

[25] Randić, M. A graph theoretical approach to conjugation and resonance energies of hydrocarbons. Tetrahedron, 1977, 33, 19051920.

[26] Klein, D.J.; Trinajstić, N. Foundations of conjugated circuit models. Pure Appl. Chem., 1989, 61, 2107-2115.

[27] Trinajstić, N. Chemical Graph Theory, CRC Press, 1992.

[28] Pauling, L. The calculation of matrix elements for Lewis electronic structures of molecules. J. Chem. Phys., 1933, 1, 280-283.

[29] Pauling, L.; Wheland, G.W. The nature of the chemical bond. V. The quantum-mechanical calculation of the resonance energy of benzene and naphthalene and the hydrocarbon free radicals. $J$. Chem. Phys., 1933, 1, 362-174.

[30] Pauling, L.; Sherman, J. The nature of the chemical bond. VI. The calculation from thermochemical data of the energy of resonance of molecules among several electronic structures. J. Chem. Phys., 1933, 1, 606-617.

[31] Pauling, L.; Wheland, G.W. The nature of the chemical bond. V. J. Chem. Phys., 1934, 2, 482-482.

[32] Simpson, W.T. On the use of structures as an aid in understanding II-electron spectra. J. Amer. Chem. Soc., 1953, 75, 597-603.

[33] Herndon, W.C. Resonance energies of aromatic hydrocarbons. Quantitative test of resonance theory. J. Amer. Chem. Soc., 1973, 95, 2404-2406.

[34] Herndon, W.C. Structure-resonance theory. A review of application to pi-hydrocarbon systems. Isr. J. Chem., 1980, 20, 270-275. 
[35] Manoharana, M.; Balakrishnarajana, M.M.; Venuvanalingama, P.; Balasubramanianb, K. Topological resonance energy predictions of the stability of fullerene clusters. Chem. Phys. Lett., 1994, 222, 95100 .

[36] Babić, D.; Trinajstić, N. Resonance energies of fullerenes with 4membered rings. Int. J. Quant. Chem., 1995, 55, 309-314.

[37] Ohkami, N.; Hosoya, H. Topological dependency of the aromatic sextets in polycyclic benzenoid hydrocarbons. Recursive relations of the sextet polynomial. Theor. Chim. Acta, 1983, 64, 153-170.

[38] He, W.; He, W. Topological properties of KHF-graphs and a proof of a one-to-one correspondence between Kekulé and sextet patterns of KHF-graphs. Theor. Chim. Acta, 1986, 70, 447-453.

[39] Ohkami, N. Graph-theoretical analysis of the sextet polynomial. Proof of the correspondence between the sextet patterns and Kekulé patterns. J. Math. Chem., 1990, 5, 23-42.

[40] Zhang, F.; Guo, X. Directed tree structure of the set of Kekulé patterns of generalized polyhex graphs. Discrete Appl. Math., 1991, 32, 295-302.

[41] Guo, X.; Zhang, F. Mathematical properties and structures of sets of sextet patterns of generalized polyhexes. J. Math. Chem., 1992, 9, 279-290.

[42] Zhang, F.; Chen, R. When each hexagon of a hexagonal system covers it, Discrete Appl. Math., 1991, 30, 63-75.

[43] Zhang, F.; Zheng, M. Generalized hexagonal systems with each hexagon being resonant. Discrete Appl. Math., 1992, 36, 67-73.

[44] Zhang, H.; Zhang, F. Plane elementary bipartite graphs, Discrete Appl. Math., 2000, 105, 291-311.

[45] Ohkami, N.; Motoyama, A.; Yamaguchi, T.; Hosoya, H.; Gutman, I. Graph-theoretical analysis of the Clar's aromatic sextet. Tetrahedron, 1981, 37, 1113-1122.

[46] Randić, M. Aromaticity of polycyclic conjugated hydrocarbons. Chem. Rev., 2003, 103, 3449-3605.

[47] Gutman, I.; Hosoya, H.; Yamaguchi, T.; Motoyama, A.; Kuboi, N. Topological properties of benzenoid systems. III. Recursion method for the sextet polynomial. Bull. Soc. Chim. Beograd, 1977, 42, 503-510

[48] Gutman, I. Topological properties of benzenoid systems. An identity for the sextet polynomial. Theor. Chem. Acta (Ber1.), 1977, 45, 309-315

[49] El-Basil S. Caterpillar (Gutman) trees in chemical graph theory, In: Advances in the Theory of Benzenoid Hydrocarbons, SpringerVerlag: Berlin, 1990, pp. 273-289.

[50] Gutman, I.; Zhang, F. On the ordering of graphs with respect to their matching numbers. Discrete Appl. Math., 1986, 15, 25-33.

[51] Wang, L.; Zhang, F.; Zhao, H., On the ordering of benzenoid chains and cyclo-polyphenacenes with respect to their numbers of Clar aromatic sextets. J. Math. Chem., 2005, 38, 293-309.

[52] Zhang, F.; Cyvin, S.J.; Cyvin, B.N. "Crowns", and aromatic sextets in primitive coronoid hydrocarbons. Monatshefte fur Chemie, 1990, 121, 421-432.

[53] Hosoya, H. Matching and Symmetry of Graphs. Comp. and Maths, Aplls., 1986, 12, 271-290.

[54] Cyvin, S.J. Resolution of Hosoya's Mystery. Bull. Chem. Soc. Jpn., 1988, 61, 4445-4446.

[55] Zhang, F.; Chen, R.; Cyvin, S.J. A complete solution of Hosoya's mystery. Acta Mathematicae Applicatae Sinica, 1996, 12, 59-63.

[56] Zhang, F.; Chen, R. A theorem concerning polyhex graphs. MATCH Commun. Math. Comput. Chem., 1986, 19, 179-188.

[57] Zhou, S.; Zhang, H.; Gutman, I. Relations between Clar structures, Clar covers, and the sextet-rotation tree of a hexagonal system. Discrete Appl. Math., 2008, 156, 1809-1821.

[58] Gutman, I. The R-polynomial: a new combinatorial technique in resonance theory. Bull. Soc. Chim. Beograd, 1981, 46, 17-22.

[59] John, P.E. Calculating the cell polynomial of catacondensed polycyclic hydrocarbons. J. Chem. Inform. Comput. Sci., 1994, 34, 357-359

[60] John, P.E.; Sachs, H.; Zheng, M. Kekulé patterns and Clar patterns in bipartite plane graphs. J. Chem. Inform. Comput. Sci., 1995, 35, 1019-1021.

[61] Shiu, W.C.; Lam, P.C.B.; Zhang, F.; Zhang, H. Normal components, Kekulé patterns, and Clar patterns in plane bipartite graphs. J. Math. Chem., 2002, 31, 405-420.

[62] Zhang, H. Perfect matchings in plane elementary bipartite graphs. Ph.D. Thesis, Sichuan University: Chengdu, 1994.

[63] Chen, Z. Directed tree structure of the set of Kekulé patterns of polyhex graphs. Chem. Phys. Lett., 1985, 115, 291-293.
[64] Zhang, H.; Zhang, F. The rotation graphs of perfect matchings of plane bipartite graphs. Discrete Appl. Math., 1997, 73, 5-12.

[65] Zhang, H.; He, J. A comparison between 1-factor count and resonant pattern count in plane non-bipartite graphs. J. Math. Chem., 2005, 38, 315-324.

[66] Bergan, J.L.; Cyvin, B.N.; Cyvin, S.J. The Fibonacci numbers, and Kekulé structures of some corona-condensed benzenoids (corannulenes). Acta Chim Hung., 1987, 124, 299-314.

[67] El-Basil, S. Clar sextet theory of buckminsterfullerene $\left(\mathrm{C}_{60}\right)$. J. Mol. Struct. (Theochem), 2000, 531, 9-21.

[68] Shiu, W.C.; Lam, P.C.B.; Zhang, H. Clar and sextet polynomials of buckministerfullerene. J. Mol. Struct. (Theochem), 2003, 662, 239248.

[69] Ye, D.; Qi, Z.; Zhang, H. On $k$-resonant fullerene graphs. SIAM J. Discrete Math., 2009, 23, 1023-1044.

[70] Sereni, J.; Stehlík, M. On the sextet polynomial of fullerenes. $J$. Math. Chem., 2010, 47, 1121-1128.

[71] Austin, S.J.; Fowler, P.W.; Hansen, P.; Manolopoulos, D.E. Zheng, M. Fullerene isomers of C60. Kekul $;$ äe counts versus stability. Chem. Phys. Lett., 1994, 228, 478-484.

[72] Zhang, H.; Ye, D.; Liu, Y. A combination of Clar number and Kekulé count as an indicator of relative stability of fullerene isomers of $\mathrm{C}_{60}$. J. Math. Chem., 2010, 48, 733-740.

[73] Zhang, H.; Ye, D. An upper bound for the Clar Number of fullerene graphs, J. Math. Chem., 2007, 41, 123-133.

[74] Gutman, I.; Cyvin, S.J. Introduction to the Theory of Benzenoid Hydrocarbons; Springer: Berlin, 1989.

[75] Gutman, I.; Babi C , D. Characterization of all-benzenoid hydrocarbons. J. Mol. Struct.(THEOCHEM), 1991, 251, 367-373.

[76] Ye, D.; Zhang, H. Extremal fullerene graphs with the maximum Clar number. Discrete Appl. Math., 2009, 157, 3152-3173.

[77] Fowler P.W.; Manolopoulos, D.E. An Atlas of Fullerenes, Clarendon Press: Oxford, 1995.

[78] Zhang, F.; Zhang, H.; Liu, Y. The Clar covering polynomial of hexagonal systems II. An application to resonance energy of condensed aromatic hydrocarbons. Chin. J. Chem., 1996, 14, 321325 .

[79] Zhang, H.; Zhang, F. The Clar covering polynomial of hexagonal systems III. Discrete Math., 2000, 212, 261-269.

[80] Zhang, $\mathrm{H}$. The Clar covering polynomial of S,T-isomers. MATCH Commun. Math. Comput. Chem., 1993, 29, 189-197.

[81] Zhang, H. The Clar covering polynomial of hexagonal systems with an application to chromatic polynomials, Discrete Math. 1997, 172, 163-173

[82] Gutman, I.; Gojak, S.; Furtula, B. Clar theory and resonance energy. Chem. Phys. Lett., 2005, 413, 396-399.

[83] Gutman, I.; Gojak, S.; Stanković, S.; Furtula, B. A concealed difference between the structure-dependence of Dewar and topological resonance energy. J. Mol. Struct. (Theochem), 2005, 757, 119-123.

[84] Gutman, I.; Furtula, B.; Balaban, A.T. Algorithm for simultaneous calculation of Kekulé and Clar structure counts, and Clar number of benzenoid molecules. Polyc. Arom. Comp., 2006, 26, 17-35.

[85] Gojak, S.; Radenković, S.; Kova C ević, R.; Stankovic, S.; Durdević, J.; Gutman, I. A difference between the $\pi$-electron properties of catafusenes and perifusenes. Polyc. Arom. Comp., 2006, 26, 197-206.

[86] Gutman, I.; Gojak, S.; Furtula, B.; Radenković, S.; Vodopivec, A. Relating total $\pi$-electron energy and resonance energy of benzenoidmolecules with Kekulé- and Clar-structure-based parameters. Monatsh. Chem., 2006, 137, 1127-1138.

[87] Gojak, S.; Gutman, I.; Radenković, S.; Vodopivec, A. Relating resonance energy with Zhang-Zhang polynomial. J. Serb. Chem. Soc., 2007, 72, 673-679.

[88] Gutman, I.; Borouićanin, B. Zhang-Zhang polynomial of multiple linear hexagonal chains. Z. Naturforsch, 2006, 61a, 73-77.

[89] Guo, Q.; Deng, H.; Chen, D. Zhang-Zhang polynomials of cyclopolyphenacenes. J. Math. Chem., 2009, 46, 347-362.

[90] Zhang, H.; Ji, N.; Yao, H. Transfer-matrix calculation of the Clar covering polynomial of hexagonal systems. MATCH Commun. Math. Comput. Chem., 2010, 63, 379-392.

[91] Chen, D.; Deng, H.; Guo, Q. Zhang-Zhang polynomials of a class of pericondensed benzenoid graphs. MATCH Commun. Math. Comput. Chem., 2010, 63, 401-410. 
[92] Herndon, W.C.; Hosoya, H. Parameterized valence bond calculations for benzenoid hydrocarbons using Clar structure. Tetrahedron, 1984, 40, 3987-3995.

[93] El-Basil, S. Combinatorial Clar sextet theory. Theor. Chim. Acta, 1986, 70, 53-65.

[94] Randić, M.; El-Basil, S. Graph theoretical analysis of large benzenoid hydrocarbons. J. Mol. Struct. (Theochem), 1994, 303, 233-245.

[95] Randić, M.; El-Basil, S.; Nikolić, S.; Trinajstić, N. Clar polynomial of large benzenoid systems. J. Chem. Inf. Comput. Sci., 1998, 38, 536-574.

[96] Randić, M. Resonance energy of very large benzenoid hydrocarbons. Int. J. Quant. Chem., 1980, 17, 549-586.

[97] Nikolić, S.; Randić,M.; Klein, D.J.; Plavšić, D.; Trinajstić, N. The conjugated-circuit model: application to benzenoid hydrocarbons. J. Mol. Struct., 1989, 198, 223-237.

[98] Trinajstić, N.; Nikolić, S.; Klein D.J. Quantum-mechanical and computational aspects of the conjugated-circuit model. J. Mol. Struct. (Theochem), 1991, 229, 63-89.

[99] Vogler, H.; Trinajstić, N. The conjugated circuits model. Theor. Chim. Acta, 1988, 73, 437-448.

[100] Nikolić, S.; Trinajstić, N.; Klein, D.J. The conjugated-circuit model. Comput. Chem., 1990, 14, 313-322.

[101] Plavšić, D.; Nikolić, S.; Trinajstić, N. The conjugated-circuits model: on the selection of the parameters. Croat. Chem. Acta, 1990, 63, 683-692.

[102] Plavšić, D.; Nikolić, S.; Trinajstić, N. The conjugated-circuit model: The optimum parameters for benzenoid hydrocarbons. $J$. Math. Chem., 1991, 8, 113-120.

[103] Klein D.J.; Seitz, W.A.; Schmalz, T.G. Conjugated-circuit computations for conjugated hydrocarbons, In: Computational Chemical Graph Theory; Rouvray, D. H., Ed.; Nova: New York, 1990; pp. 127-147.
[104] Plavšić, D.; Trinajstić, N.; Randić, M.; Venier, C. The conjugatedcircuit model: application to benzenoid hydrocarbon radicals. Croat. Chem. Acta, 1989, 62, 719-740.

[105] Guo, X.; Randic, M. Recursive method for enumeration of linearly independent and minimal conjugated circuits of benzenoid hydrocarbons. J. Chem. Inf. Comput. Sci., 1994, 34, 339-348.

[106] Randic', M.; Guo, X. Generalized bond orders, Int. J. Quant. Chem., 1994, 49, 215-237.

[107] Guo, X.; Randć, M. Recursive formulae for enumeration of $L M$ conjugated circuits in structurally related benzenoid hydrocarbons. J. Math. Chem., 2002, 30, 325-342.

[108] Guo, X.; Randić, Klein, D.J. Analytical expressions for the count of $L M$-conjugated circuits of benzenoid hydrocarbons. Int. J. Quant. Chem., 1996, 60, 943-958.

[109] Guo, X. $k$-resonance in benzenoid systems, open-ended carbon nanotubes, toroidal polyhexes; and $k$-cycle resonant graphs, MATCH Commun. Math. Comput. Chem., 2006, 56, 439-456.

[110] Shiu, W.C.; Zhang, H. A complete characterization for $k$-resonant Klein-bottle polyhexes. J. Math. Chem., 2008, 43(1), 45-59.

[111] Zhang, H.; Ye, D. k-resonant toroidal polyhexes. J. Math. Chem., 2008, 44, 270-285.

[112] Liu, S.; Zhang, H. Maximally resonant polygonal systems. Discrete Math., 2010, 310, 2790-2800

[113] Shiu, W.C.; Zhang, H.; Liu, S. Maximal resonance of cubic bipartite polyhedral graphs. J. Math. Chem., 2010, 48, 676-686.

[114] Zhang, H; Liu, S. 2-resonance of plane bipartite graphs and its applications to boron-nitrogen fullerenes. Discrete Appl. Math., 2010, 158, 1559-1569.

[115] Li, Q.; Liu, S.; Zhang, H. 2-extendability and $k$-resonance of nonbipartite Klein-bottle polyhexes. Discrete Appl. Math., 2011, 159 , 800-811.

(C) Zhang et al.; Licensee Bentham Open.

This is an open access article licensed under the terms of the Creative Commons Attribution Non-Commercial License (http://creativecommons.org/licenses/by-nc/3.0/) which permits unrestricted, non-commercial use, distribution and reproduction in any medium, provided the work is properly cited. 